\title{
Sangue mestruale e latte materno: riflessioni e nuove proposte. Intorno all'allattamento nella Grecia antica*
}

\author{
Giulia Pedrucci
}

\section{Summary}

Within a larger study on breast-feeding in ancient Greece, we dwelt on four subjects (the superstitions concerning menstrual blood, milk and dairy products consumption by the Athenians, different kinds of milk and beliefs related to the transmission of hereditary characteristics through human milk, the connection between milk, breast and madness) on which we have identified a certain number of neglected sources. Starting from these, we can gain not only some mosaic tiles of the overall fragmentary view on habits and beliefs about breast-feeding, but also, more generally, helpful hints on some aspects of the Greek world and mentality that we barely know. In attempting to reach some general conclusions, we have also considered the iconographic sources, trying to explain, in part at least, the reason for the almost complete absence of scenes of breast-feeding in the archaic and classical art.

Keywords: Breast-feeding, ancient gynecology, menstrual blood, human milk, dairy products

\footnotetext{
* Desidero ringraziare sentitamente la Prof.ssa Véronique Dasen, con la quale ho avuto il piacere e l'onore di collaborare durante una borsa di studio post-dottorato all'Université de Fribourg, e grazie alla quale il mio lavoro è significativamente progredito. Senza il suo aiuto e la sua squisita disponibilità questo articolo non avrebbe mai visto la luce. Articolo che rappresenta una piccola parte della mia tesi di dottorato, per cui non posso dimenticare di ringraziare chi ha seguito, con passione e competenza (e pazienza) consuete, il mio lavoro sin dalle più lontane origini: un grazie sincero e affettuoso al Prof. Dario M. Cosi.

Per la traduzione dei passi tratti dal Corpus Hippocraticum si preferisce utilizzare, sebbene in francese e tutt'altro che recente, l'edizione di Emile Littré (Paris 1861, ripr. Amsterdam 1973). Le traduzioni dei passi dell'Orestea di Eschilo sono di Vincenzo Di Benedetto, Milano 1995.
}

Giulia Pedrucci (Ph.D. Dr), Université de Fribourg, Institut des sciences de l'Antiquité et du monde byzantin, Rue Pierre-Aeby 16, CH-1700 Fribourg; Università di Bologna, Dipartimento di Storia Culture Civiltà, Via Zamboni, 38, I-40126 Bologna (giulia.pedrucci@unibo.it). 


\section{Riassunto}

All'interno di un più ampio studio sull'allattamento nella Grecia antica, ci siamo soffermarti sopra quattro argomenti (i pregiudizi connessi con il angue mestruale; il consumo di latte e latticini da parte degli Ateniesi; i diversi tipi di latte e le eventuali credenze relative alla trasmissione di caratteri ereditari attraverso il latte umano; il nesso fra latte, seno e follia) in merito ai quali abbiamo individuato alcune fonti (in parte neglette) da cui è possibile non soltanto ricavare tasselli da aggiungere al frammentario mosaico delle abitudini e delle credenze relative, ma anche, più in generale, elementi che possono aiutare sia a riflettere sopra alcuni aspetti del mondo greco e della mentalità greca che conosciamo poco sia ad approfondirne e arricchirne altri. Nel tentativo di arrivare ad alcune conclusioni generali, si sono prese in considerazione anche fonti iconografiche, cercando di spiegare, almeno in parte, le ragioni della quasi totale assenza di scene di allattamento nell'arte di epoca arcaica e classica.

\section{Introduzione}

Si tende, soprattutto quando si parla di ginecologia antica, troppo spesso a mescolare, con eccessiva disinvoltura, le fonti greche di epoca arcaica e classica con quelle di epoca imperiale. La documentazione, in realtà, risulta essere molto diversa sia qualitativamente che quantitativamente, soprattutto per quanto concerne l'allattamento materno. Da un lato, infatti, abbiamo il fiume di parole scritto dai medici di epoca imperiale, principalmente in relazione al costume di utilizzare balie da latte, dall'altro il silenzio quasi totale degli autori greci di epoca classica. Ma, scandagliando quasi «chirurgicamente» la documentazione greca (non soltanto medica), si possono rintracciare alcune significative informazioni, per quanto isolate e spesso decontestualizzate, che ci forniscono importanti elementi da aggiungere al frammentario quadro delle abitudini e delle credenze relative all'allattamento nella Grecia antica.

Asserire, come risulta implicito dalle nostre parole, che non esiste un mondo greco-romano non significa, però, in nessun modo asserire che i due mondi non possono comunicare, fornendoci tasselli utili per ricostruire quel grande mosaico che è per noi il mondo antico, che sia greco, che sia romano, pur restando separati.

Quest'ultimo punto introduce il ben noto problema metodologico relativo all'uso delle fonti primarie e delle fonti secondarie e, in particolare, al rap- 
porto fra fonti dirette e fonti frutto di citazione, questione che diventa straordinariamente complessa se le fonti appartengono a due epoche o a due culture differenti, soprattutto nel caso in cui si abbia a che fare con fonti appartenenti ad una cultura diversa e, nella forma che a noi interessa, successivamente sviluppatasi (quella romana rispetto a quella greca, nella fattispecie), ma con la quale esistono rapporti diretti, analogie, continuità,o, meglio, risemantizzazioni (nelle teorie mediche e ginecologiche, a esempio).

Con ogni evidenza non è possibile né tantomeno pensabile proporre soluzioni univoche e «universali» a problematiche metodologiche di così ampia portata, di conseguenza l'unica strada percorribile è, a nostro avviso, quella dell'analisi caso per caso. Possiamo, allora, usare un testo di Plinio oppure un testo di Sorano per cercare di ricostruire, di ipotizzare credenze analoghe nel mondo greco? Considerando la coerenza e il carattere tendenzialmente conservativo - nel loro impianto generale - delle teorie ginecologiche nel mondo antico e considerando la coerenza e la verosimiglianza delle ricostruzioni ipotizzate rispetto a un meglio noto quadro di insieme, con le dovute cautele ci pare che la risposta possa essere positiva. Questo non significa - come già evidenziato - in nessun modo affermare l'esistenza di un unico mondo greco-romano, né appiattire le differenze, né tantomeno ritenere un'operazione di questo tipo sempre possibile e/o auspicabile. Utilizzare, a esempio, Sorano per sostenere che le donne greche di epoca arcaica e classica non allattavano, per contro non ci pare corretto, dal momento che abbiamo un numero relativamente elevato di fonti greche che sembrano testimoniarci il contrario (e, d'altronde, gli stessi autori latini ci informano che anche a Roma non era sempre stato così).

Un'ulteriore precisazione è necessaria: trattandosi di storia antica, il «lusso» di avvalersi soltanto di documentazione scritta è mera utopia. L'essenziale è, a nostro avviso, cercare di fare «dialogare» fonti scritte e fonti non scritte, ma senza ritenere le une il fedele specchio delle altre, e viceversa. Silenzio (e, ancor di più, assenza) possono valere di più di mille parole, se opportunamente contestualizzati. Superfluo sottolineare che il rischio è alto, dal momento che silenzio e assenza possono essere soltanto fittizi, cioè dovuti alla perdita del materiale. In quel «campo favorevole per ciarlatani» che è la storia antica non dimentichiamo che «scarsi documenti significano peggiore storia, ma non escludono qualche forma ipotetica di storia». ${ }^{1}$

Il quadro di insieme che cercheremo di ricostruire è certamente pieno di diseguaglianze e di lacune, ma non per questo privo di una sua importanza e di una sua dignità scientifica. Pur non dimenticando quanto sia ardito e cri- 
ticabile basare conclusioni anche su ipotesi di lavoro e su pericolosi argumenta ex silentio, siamo profondamente persuasi che una luce incerta sia meglio del buio. ${ }^{2}$

\section{La natura del latte}

Nei trattati medici antichi troviamo idee piuttosto precise circa la natura del latte umano, considerato un succedaneo del sangue, con cui il feto è stato nutrito durante la gravidanza e che, prima della gestazione, veniva periodicamente espulso dal corpo femminile grazie al ciclo mestruale. La credenza che il latte umano altro non fosse che sangue dalle mutate caratteristiche risale probabilmente al pensiero presocratico, ${ }^{3}$ ma viene elaborata principalmente da Aristotele, che ci offre spiegazioni assai particolareggiate del processo che avviene all'interno del corpo femminile ( $G A$, IV 8, 776a-776b) ${ }^{4}$ Le secrezioni di natura ematica, costituenti nell'uomo il seme e nella donna il sangue mestruale, se uniti con la fecondazione, producono, grazie all'impulso generatore dell'elemento maschile, una materia attiva che serve sia per la formazione dell'embrione sia per il suo nutrimento, destinato all'uno e all'altro uso in quantità variabili secondo le diverse fasi della gestazione. Lo Stagirita afferma, infatti, che la stessa materia procura nutrimento e costituisce la base naturale della generazione, specificando subito dopo che il latte è sangue cotto, ma non corrotto. ${ }^{5}$ Dalle parole del filosofo si può dedurre che il latte umano è un'ulteriore trasformazione, per cottura, ${ }^{6}$ di quel sangue mestruale che, modificandosi sotto l'impulso del seme maschile, ha dato prima origine all'embrione, poi al suo nutrimento intra-uterino. Le teorie aristoteliche, assai simili nella sostanze a quelle presenti nel Corpus Hippocraticum, riescono, fra le altre cose, a spiegare l'assenza delle mestruazioni durante la gravidanza e durante l'allattamento: secondo natura - egli scrive in $G A$, IV 8, 777a - non vengono mestruazioni alle donne che allattano né le donne che allattano possono restare incinte, dal momento che, nel caso di una successiva gravidanza, il latte scompare, essendo la natura

1 Momigliano 1980,15s. Lo storico sottolinea l'importanza di avanzare comunque ipotesi interpretative per capire la storia.

2 «An uncertain light is better than none»: Dodds 1951, VII.

3 Emp., 31B68 Diels-Kranz (riportato anche da Aristotele in GA, IV 8, 777a, v. n. 5). Cf. n. 51.

4 Danese 1997, 45-50. Sull'argomento si veda anche Bodiou 2006, 164-66.

$5 G A$, IV 8, 777a. Il medesimo concetto è espresso più volte nel IV Libro, come, a esempio, in GA, IV 1, 766a. Cf. n. 52.

6 V.n. 9. 
del latte e quella dei mestrui la stessa. ${ }^{7}$ Tutto ciò è riassunto in maniera

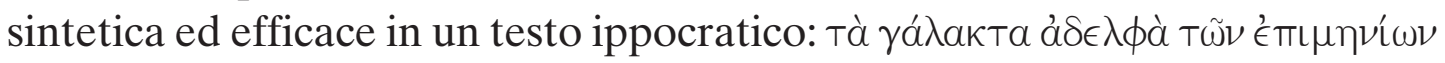
(Epid., II 17, Littré V 118). «Le lait, frère des règles».

Le teorie dei medici e dei filosofi greci trovano conferma, in linea di massina, negli autori di epoca romana ${ }^{8}$ per gli scrittori greci e latini-riassumendo brutalmente - il latte umano è senza dubbio il sangue mestruale della donna, che, grazie all'impulso e al calore del seme maschile, prima ha formato e nutrito il feto, poi, in seguito a una successiva cottura, ${ }^{9}$ è diventato il bianco alimento con cui viene nutrito il neonato (non necessariamente il figlio).

Vorremmo soffermarci su quattro argomenti, relativamente ai quali, a nostro avviso, esistono fonti, forse un po' trascurate, che ci permettono di avanzare «riflessioni e nuove proposte» per quanto concerne l'allattamento nella Grecia antica: i pregiudizi connessi con il sangue mestruale; il consumo di latte e latticini da parte degli Ateniesi; i diversi tipi di latte e le eventuali credenze relative alla trasmissione di caratteri ereditari attraverso il latte umano (sia nelle fonti mediche che in quelle mitologiche); il nesso fra latte, seno e follia. Nel tentativo di arrivare ad alcune conclusioni generali, ci si soffermerà anche sulle fonti iconografiche. Siamo ben consapevoli della difficoltà di tenere uniti argomenti così diversi, nonostante la presenza di un motivo unificante (la natura del latte e l'allattamento), ma lo scopo del presente lavoro vuole «soltanto» essere quello di offrire nuovi spunti di riflessione a chi, con maggiore competenza di noi, si occupa abitualmente di storia della medicina antica. In nome dell'interdisciplinarietà.

7 In $H A$, VII 3, 583a, Aristotele spiega più chiaramente il processo che avviene all'interno del corpo femminile: trascorsi alcuni giorni dal concepimento non si hanno più mestruazioni secondo la naturale normalità e il residuo di sangue sale alle mammelle, dove diventa latte. Per quando concerne il Corpus Hippocraticum, gli argomenti in questione sono trattati principalmente nel De natura pueri. Si vedano: Nat. puer., 14 (Littré VII 492), 21 (Littré VII 510s.); Aph., V 60s. (Littré IV 554); Mul., I 25 (Littré VIII 64). In alcuni casi le mestruazioni «chiuse» all'interno del corpo possono trasformarsi in latte senza che la donna sia incita, a conferma del legame connaturale fra mestruo e latte materno: Aph., 39, 50 (Littré IV 544, 550). Notiamo che, secondo l'autore del De natura pueri, il latte è già stato trasformato quando risale dal ventre, mentre, secondo l'autore del De glandulis, il latte si trasforma nei seni. Ritroviamo, sia nell'opera aristotelica sia nel Corpus Hippocraticum, la medesima teoria del riscaldamento del seme all'interno dell'utero: Nat. puer., 12 (Littré VII 486s.). Per quanto concerne l'apporto maschile e quello femminile al concepimento, i medici dei trattati ippocratici riconoscono un ruolo più «attivo» alla donna, v.: Gland., 16 (Littré VIII 572-74; Epid., II 17 (Littré V 116); Alim., 37 (Littré IX 110).

8 Varr., Catus de liberis educandis, fr. 8 Riese; Sor., Gynaec., II 22; Gal., VI 45 K.; Orib., Inc., XXX 3, 121 Raeder; Plut., 32, 495 e-f; Favorino, fr. 38 Barigazzi (riportato da Gell., Noct. Att., XII 1.1-23). Per l'analisi di queste fonti si rimanda a Danese 1997.

9 «Le corps des femmes est une cuisine»: Bodiou 2006, 162s. Cf. DuBois 1990, 147-74. Lo sbiancamento dovuto al riscaldamento, stando all'opinione di Clemente Alessandrino, che conosce e condivide la teoria dell'emogenesi del latte umano (Paed., I 39-41), eviterebbe al bambino lo spavento di fronte alla visione del sangue. 


\section{Pregiudizi connessi con il sangue mestruale}

In questo paragrafo si cerca di dimostrare che anche in Grecia, come a Roma, esistevano radicati pregiudizi contro il sangue mestruale. Per quanto quest'affermazione possa sembrare scontata, si è spesso cercato si sostenere, almeno in parte, il contrario. ${ }^{10} \mathrm{Si}$ utilizzeranno a questo scopo fonti secondarie e indirette.

Non è necessario soffermarsi troppo a lungo sui pregiudizi, ben radicati e longevi, relativi al sangue mestruale. È necessario, però, sottolineare che essi emergono con più vigore nei testi degli autori latini rispetto ai testi degli autori greci. Plinio, in particolare, ci offre un'ampia gamma di esempi relativi alla potenza malefica delle mestruazioni. Nihil facile reperiatur mulierum profluuio magis monstrificum: non occorre aggiungere molto! ${ }^{11}$

Non mancano, però, contrariamente a quanto è stato frequentemente sostenuto, ${ }^{12}$ accenni a credenze simili nei testi greci. Ci riferiamo in particolare a due testimonianze aristoteliche. La prima, molto problematica, si trova all'interno del breve trattato intitolato Sui sogni. Relativamente a essa si è brillantemente espressa Françoise Frontisi-Ducroux, facendo riferimento al significato dello specchio nel mondo greco, oggetto esclusivamente femminile. ${ }^{13}$ Aristotele scrive, in relazione agli effetti che le mestruazioni hanno sugli specchi:

10 È particolarmente nota la tesi - peraltro non nuova - della Dean-Jones 199 (cf. King 1998, 88), la quale sostiene che le mestruazioni femminili non avrebbero costituito un «tabù» nella Grecia di epoca classica, mentre lo sarebbero divenute nell'età alessandrina e nel periodo romano. La studiosa prende in considerazione diversi aspetti: il silenzio relativo alle mestruazioni nelle fonti greche che non siano «scientifiche» (trattati medici, biologici); l'assenza di miti concernenti le mestruazioni; la mancanza di «cultural taboos» riguardanti le mestruazioni nella Grecia classica. Fra i diversi argomenti che dimostrano la debolezza di questa tesi, ci sembra pertinente osservare che il silenzio può, da un lato, indicare l'assenza di un «tabù», ma, dall'altro, può indicare l'esatto opposto, cf. n. 20.

11 Plin., NH, VII 13.64. Vediamo l'intero passo: «Ma non sarebbe facile trovare qualcosa di più prodigioso del flusso mestruale delle donne. Al sopraggiungere di una donna che ha le mestruazioni il mosto inacidisce; al suo contatto le messi diventano sterili; muoiono gli innesti, bruciano i germogli dei giardini, cadono i frutti degli alberi presso cui la donna si è fermata; al suo solo sguardo, la lucentezza degli specchi si appanna, si smussa la punta delle lame, si oscuro lo splendore dell'avorio, muoiono le api negli alveari; persino il bronzo e il ferro si arrugginiscono all'istante e il bronzo prende un odore sgradevole» (Trad. G. Ranucci, Torino 1983). In XXVIII 23.77-85, Plinio insiste di nuovo sulla potenza malefica delle mestruazioni, che possono tuttavia essere utilizzate come insetticida contro i bruchi e altri insetti campestri; per questa ragione si vedono spesso le donne della Cappadocia percorrere i campi con il vestito alzato sulle natiche. Venendo in contatto con una donna mestruata, il filo del rasoio si smussa, il cuoio prende un odore fetido e si copre di ruggine, soprattutto se la luna è in fase calante, soltanto per ricordare alcuni fenomeni. Il sangue mestruale può inoltre provocare l'aborto nelle giumente e anche nelle donne gravide, v.: XXVIII 20.70, 22.79 .

12 V.n. 10.

13 Frontisi-Ducroux/Vernant 2003, 119-21. 
Da questi «specchi» risulta al tempo stesso evidente che la vista come subisce una certa affezione così anche ne produce. Nel caso degli specchi particolarmente lustri, infatti, quando le donne al tempo delle mestruazioni guardano nello specchio, sulla superficie dello specchio stesso si genera come una nuvola sanguigna: se lo specchio è nuovo, non è facile pulire la macchia siffatta, se invece è vecchio, è facile. La causa [...] è che non soltanto la vista subisce una certa affezione da parte dell'aria ma anche ne produce una e muove, come fanno anche le cose splendenti [...] Gli occhi si trovano dunque ragionevolmente nella medesima posizione in cui è qualunque parte del corpo, quando vi sono le mestruazioni, giacché per natura sono venosi. Pertanto quando si generano le mestruazioni, a causa del perturbamento e dell'infiammazione del sangue, la differenza negli occhi non ci è chiara ma c'è (la natura del seme e delle mestruazioni, infatti, è la stessa): l'aria ne viene mossa, e l'aria che sta sullo specchio, trovandosi a continuazione, la rende tale quale l'affezione che essa subisce. Questa «produce» l'apparizione dello specchio. In questo modo gli indumenti molto puliti si sporcano velocemente... ${ }^{14}$

Per dimostrare che gli organi dei sensi percepiscono differenze minime, Aristotele fa appello all'esempio degli specchi. Ci si aspetterebbe che egli assimilasse lo specchio a un organo visivo, ma, non condividendo la teoria della visione per riflesso di Democrito, Aristotele spiega che la vista percepisce piccole differenze, come le modificazioni del corpo femminile in periodo di disturbo o infiammazione, e che, essendo sia attiva che passiva, trasmette a sua volta ciò che subisce allo specchio, che agisce come un rivelatore di ciò che la vista ha subito.

Il fenomeno degli specchi appena descritto è soltanto una delle numerose testimonianze della netta cesura tra mondo femminile e sguardo maschile, e, come abbiamo visto ricordando il passo di Plinio, ${ }^{15}$ lungi dall'essere prerogativa del solo Aristotele, fa parte di una ricca serie di credenze relative al ciclo mestruale nel mondo antico, dalle quali risultano gli effetti del ciclo femminile in relazione a tre distinti piani sensoriali: la vista, appunto, il contatto e l'olfatto.

Sia dalle parole di Aristotele che da quelle di Plinio si intuisce chiaramente, inoltre, la relazione tra il mestruo e la fecondità. Il corpuscoli visivi che emanano dagli occhi, e, conseguentemente, dal corpo femminile, sono impregnati di sangue durante il periodo delle mestruazioni, ma restano invisibili e soltanto lo specchio li rende visibili. Per Aristotele, come per Plinio, il periodo immediatamente successivo al ciclo è considerato il momento più favorevole per concepire, ${ }^{16}$ dal momento che il concepimento risulta

$14 S V, 459$ b (trad. A.L. Carbone in Aristotele. L'anima e il corpo. Parvia Naturalia, Milano 2002). Il testo prosegue, facendo riferimento anche agli effetti di quest'aria «contaminata» sul bronzo, il quale, essendo levigato, risulta particolarmente reattivo a ogni tipo di contatto. Plinio segnala altri effetti delle mestruazioni sul bronzo, v. n. 11 .

15 V.n. 11.

16 GA, I 19, 727b; HA, VII 2, 582b. Cf. Plin., NH, VII 16.67 (secondo Plinio anche il periodo immediatamente precedente al ciclo è propizio). La Frontisi-Ducroux, in realtà, afferma che, secondo gli autori antichi, il periodo mestruale è quello più favorevole alla riproduzione. 
dall'unione del sangue femminile con lo sperma maschile. In questo modo, ciò che lo specchio rende visibile, sotto l'influsso degli sguardi insanguinati, non è altro che lo stato della matrice femminile pronta a ricevere l'impronta maschile. ${ }^{17}$

La seconda asserzione aristotelica che ci pare significativa è la seguente: le mestruazioni, per la mancata cozione, sarebbero un fenomeno simile alla diarrea $(G A, \mathrm{I} 20,728 \mathrm{a}) .{ }^{18}$ Il sangue mestruale non è che un residuo: utile, a differenza di altri residui-scarti come escrementi e urina, anzi, indispensabile, causa materiale dell'essere umano, sostrato necessario alla generazione; esso è tuttavia un prodotto in eccesso e di scarto.

A conferma di un atteggiamento né neutro, né indifferente, nei confronti del sangue mestruale possiamo considerare le testimonianze relative all'impiego di esso - e del latte umano, come vedremo nel prossimo paragrafo a fini terapeutici. La testimonianza, riportata da Plinio, ${ }^{19}$ è, purtroppo, soltanto indiretta, ma straordinariamente interessante in quanto coinvolge

17 La Frontisi-Ducroux in nota menziona una tradizione serba in base alla quale la sposa si reca in chiesa, al fine di avere figli, con uno specchio sul seno. V. Frontisi-Ducroux/Vernant 2003, 122, n. 10.

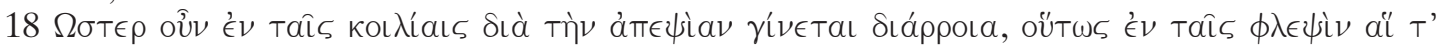

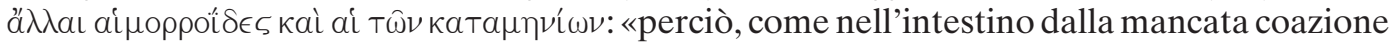
si produce la diarrea, così nelle vene i risultati sono sia le altre emorragie, sia i mestrui» (trad. D. Lanza, Torino 1971). Che mestruo e diarrea fossero, in un certo senso, messi sullo stesso piano, in quanto entrambi residui del corpo umano non perfettamente cotti e perciò da espellere, è confermato poche righe sopra, dove si legge - in riferimento però ai maschi - che in coloro che non sono più in grado di generare talvolta si ha diarrea perché il residuo, non potendo giungere a cozione e diventare sperma, si secerne nel ventre. Von Staden 1992, 8-13, ha osservato che nel mondo greco il giudizio riguardo all'utilizzo degli escrementi era negativo e che queste sostanze erano considerate altamente ripugnanti e che, nell'ambito della cosiddetta farmacopea immonda ippocratica, le feci venivano prescritte soltanto per curare le donne e, a parte un paio di casi, proprio per disturbi della matrice. Si noti, per inciso, che, fra le femmine di tutti gli animali, la donna è quella con le mestruazioni più abbondanti, v. n. 26. Cf. Tognazzi 2008. Teniamo presente, a questo proposito, il disgusto e il fastidio espresso soprattutto nella commedia aristofanea per i genitali femminili. In ben tre commedie (Eq., 1280-83; Pax, 883-85; Ve., 1280-83) il commediografo attacca violentemente tal Arifrade (identificato sia come un «collega» sia come un politico «progressista»), etichettandolo come inventore di turpi pratiche erotiche, descritte con crudo realismo, fra cui il cunnilinctus. Come osserva von Staden 1992, 13, dalle parole del coro in Eq., 1280-83, si intuisce che le secrezioni femminili sarebbero particolarmente laide e «contaminanti». Cf. Gal., XII 249 K., in cui l'autore afferma di provare maggiore disgusto

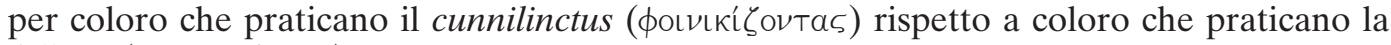

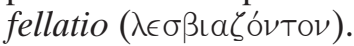

19 Plinio, invece, fa spesso riferimento all'uso di sangue mestruale a scopi curativi, v., a titolo di esempio, NH, XXVIII 22.82s. Cf. Gourevitch 1984, 101s. Si parla in particolare di rimedi per curare la gotta: la Gourevitch a questo proposito nota un rapporto, «mal explicité mais certain» fra la smodatezza sessuale e la gotta: «il est intéressant de voir cette maladie moralement suspecte guérie précisément par le liquide qui conditionne la vie sexuelle de la femme». La medesima malattia può essere curata anche, secondo Democrito, con un miscuglio di cenere ricavata dalla coscia o dal piede sinistro del camaleonte e dal latte di scrofa, ma Plinio, che riporta la notizia, la critica sarcasticamente (NH, XXVIII 29.116). 
un animale culturalmente assai intrigante: il cane. Esiste, infatti, un singolare rapporto fra cane e mestruo. Plinio afferma che, per guarire dalla rabbia, è necessario portare un braccialetto di lana di ariete nero o un lembo di stoffa impregnato di sangue mestruale, ${ }^{20}$ entrambe pratiche attribuite ad ostetriche greche, di cui una, Laide, contemporanea di Alcibiade. Come spiega lo stesso autore, si tratterebbe di un rimedio «simpatico» alla «greca», dal momento che è il sangue mestruale a causare la rabbia nei cani che lo assaggiano. ${ }^{21}$ Dettaglio ancora più singolare: i cani che assaggiano il latte di una donna che ha partorito un maschio non contraggono più la rabbia. ${ }^{22}$

$20 \mathrm{NH}$, XXVIII 22.82 (in questo caso il medesimo rimedio è indicato anche per guarire dalle febbri terzana e quartana), 22.84. Cf. Gourevitch 1984, 98s. Il filo di lana torna spesso in contesti che riguardano «cose di donne», come, a esempio, nel caso di amuleti di galattite per favorire la lattazione da portare al collo, appesi a un filo di lana di pecora gravida, v. Dasen 2003, 157. Il corpo stesso della donna è paragonato, all'interno del Corpus Hippocraticum, a un filo di lana grezzo: Mul., I 1, Littré VIII, 10s.; Gland., 16, Littré VIII, 572. Siamo di fronte a un dettaglio meritevole di successivo approfondimento, che assai verosimilmente andrà messo in relazione con il valore simbolico della tessitura nel mondo antico e con la sua relazione con la fecondità. Si veda anche Plin., NH, XXVII 29.3. Sull'argomento: Frontisi-Ducroux/Vernant 2003,121; Gualerzi 2007, 145 (l'autore ricorda in particolare come fosse diffuso il timore che le donne riunite a tessere, attività esclusivamente femminile e preclusa all'uomo, potessero tramare fra di loro inganni, complotti, addirittura assassinî). Apriamo un breve parentesi sulle due donne che, secondo Plinio, avrebbero tramandato questa ricetta: Laide e Salpe. La prima è nominata anche per un altro rimedio a base di sangue mestruale assieme a Elefantide (con questo nome è vissuta verso la fine del I secolo a.C. una poetessa che scriveva di argomenti erotici). Bettini 1998, 290-93, identifica queste donne come levatrici, nell'ambito di un'appassionante riflessione sulla figura, assai ambigua, della «levatrice-strega», cf. n. 63. Il fatto che la medicina ippocratica «ufficiale» non parli di certi rimedi a base di sangue mestruale potrebbe non essere un valido motivo per escluderne l'esistenza, cf. n. 10.

$21 \mathrm{NH}$, VII 15.64; XXVIII 22.84. Per la storia della rabbia come patologia, v. Théodoridés 1986 (in particolare 17-39 per il mondo antico). La prima attestazione di questa malattia è nell'Iliade (IX 299), secondo Celio (V secolo d.C.) era conosciuta da Democrito (V secolo a.C.), ma mancano attestazioni di essa nel Corpus Hippocraticum. Ce ne parla, però, Aristotele (HA, VIII 22. Per l'interpretazione di questo passo, un po' ambiguo, v. Théodoridés 1986, 27) ed è ben nota alla cosiddetta Scuola di Alessandria. Plinio ( $N H$, XXIX 32) attribuisce la causa della malattia ad un verme presente sulla lingua del cane, che i Greci chiamavano lytta: l'idea di un'origine parassitaria della rabbia è ancora attestata nel XIX secolo. Più spesso, però, si parla di un «veleno» come causa di questa malattia (Dioscoride, Filumeno etc., v. Théodoridés 1986, 29, 34). Filumelo (CMG X 1, p. 71), medico greco del III secolo d.C., fra i rimedi introduce il latte, come alimento che genericamente indebolisce l'azione di un veleno nel corpo. È assai singolare la menzione del latte di lepre. Essendo il virus della rabbia identificato con una sostanza velenosa, è possibile guarire il malato estraendo oppure purgando il sangue (a esempio con un cataplasma di sale). Théodoridés 1986, 22, ipotizza l'esistenza di una rappresentazione mitica della rabbia canina: Hecate. L'associazione è interessante: dea arcaica, sfuggente e polisemica, benevola verso gli uomini e kourotrophos, con un legame peculiare con il parto e quindi con il mondo muliebre, allo stesso tempo è anche inquietante per le sue abitudini notturne e il suo seguito di cagne. V. Pedrucci 2013, 129.

$22 \mathrm{NH}$, XXVIII 21.75. Plinio allude forse all'usanza, descritta da Sorano (II 8), di far succhiare il latte in eccesso delle nutrici ad altri bambini o ad animali, per evitare di dare al poppante del latte non fresco. Plutarco (46, 640f), a questo proposito, però afferma che bisogna dare a una donna con latte in eccesso un altro bambino da nutrire (per non sprecare il latte? Per 
Se è vero che, secondo una celebre definizione di Claude Lévi-Strauss, gli animali sono «bons à penser» ${ }^{23}$ cioè buoni per pensare o da pensare, il cane rappresenta in questo caso un esempio straordinariamente efficace e da approfondire.

Nell'immaginario greco la storia del contatto simbolico tra cane e natura femminile comincia con Pandora (Hes., Op., 67), progenitrice e primo esem-

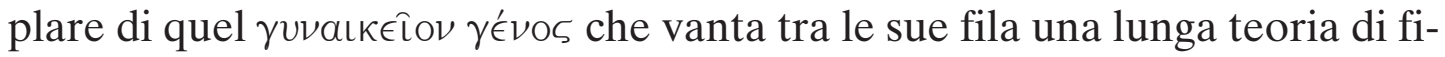
gure dai tratti canini. Il dono di un'indole canina fatto da Hermes alla prima donna è un dono indiscutibilmente infido, se si pensa a quanto spesso il cane nella Grecia antica compaia in immagini negative; questo dato contrasta, però, con le testimonianze, altrettanto numerose, che descrivono il cane come un animale assai apprezzato e presente nella vita quotidiana. ${ }^{24}$

aiutare chi ne ha meno? O, forse, per evitare che si acculi troppo latte dentro le mammelle, rischiando che vada a male?). Presso una popolazione nella Valle dello Yaghnob in Tajikistan è documentata l'usanza, basata su principi «simpatici» (v. n. 46), di allattare un cucciolo di cane per trasmettere il malocchio a madri che hanno già perso più figli, v. Delaini 2011, 162 (per le popolazioni di origine iraniana il cane è un animale sacro). I cuccioli di cane, però, a nostro avviso potevano essere utilizzati anche in caso di ragadi congiunte a mastiti, per aiutare la donna nella guarigione (in caso di ragadi sanguinanti il bambino non può succhiare; in tempi ancora relativamente recenti, soprattutto nelle zone rurali, esistevano donne capaci di succhiare il latte senza usare i denti che venivano chiamate per aiutare le donne affette da simili problemi). Plinio descrive un altro singolare utilizzo dei cani lattanti (catulus lactantes), che venivano applicati alle viscere doloranti: essi in questo modo «assorbivano» su di loro il male e conseguentemente morivano. Era necessario seppellire secondo le norme religiose i cuccioli morti in questo modo ( $N H$, XXX 14.42-43). Un analogo contagio di tipo simpatico è descritto ancora da Plinio (NH, XXX 20.64) e da Marcello di Bordeaux (XXVII 132. Cf. XXI 1, molto simile al precedente passo pliniano): per diagnosticare e curare malattie interne è necessario far coricare un cucciolo (catulus, che ancora non vede, secondo Plinio), al quale il paziente deve dare da bere latte dalla sua bocca con frequenza. Dopodiché è necessario aprire il ventre dell'animale (e, secondo Plinio, seppellirlo). Questa pratica è analizzata da Mauss 1965, 65, e potrebbe spiegare un ritrovamento come quello di Lugnano in Teverina, dove sono stati scavati, in un cimitero databile alla metà del V secolo d.C., 47 tombe di bambini (feti, neonati, un bambino di circa 2-3 anni) e gli scheletri di almeno 12 cani di età inferiore ai 6 mesi e quello di un cane di un anno (v. De Grossi Mazzorin/ Minniti 2010, 58): il cane si conferma un animale culturalmente affatto neutro. Vedremo, inoltre, che, secondo Plinio, il latte prodotto per un maschio è più efficace di quello prodotto per una femmina, v. n. 61.

23 Lévi-Strauss 1991, 126.

24 La Franco 2003, muovendo da tale apparente contraddizione, individua l'origine dell'insulto «canino» nella contiguità tra animale e uomo. Il cane partecipa al rito sociale dell'alimentazione; responsabile delle proprie azioni, consapevole del patto di collaborazione, rappresenta, come animale metonimico, il suo padrone, ma è anche incline a molte debolezze. Primo tra suoi simili, ultimo tra gli uomini, diviene emblema della mancanza di ritegno proprio perché è l'unico animale cui si richieda di averlo. Si noti un dettaglio importante: non bisogna vedere nella «cagneria» al femminile il rimando a un referente animale femmina. Come scrive la Franco 2003, 206, la distinzione riguarda il comparandum e non il comparatum. Soltanto per un effetto contestuale prodotto dall'uso italiano Agamennone, a esempio, è «cane», mentre Elena è «cagna»: in greco essi sono entrambi кúveऽ e basta. Per un interessante parallelo fra cani, cavalli, donne e schiavi nell'opera di Senofonte, v. Vilatte 1986: i migliori di tutte e quattro le «categorie» menzionate possono riuscire a eccellere, se ben educati, fino ad assomigliare al loro padrone. 
Nell'associazione con le Erinni, in particolare, si evidenzia un'attrazione del cane verso il sangue, che contribuisce alla connotazione, per molti aspetti negativa, del fedele amico dell'uomo. Vorremmo, a questo proposito, sottolineare una caratteristica che, fra gli animali domestici, appartiene soltanto alla cagna ${ }^{25}$ la presenza di perdite vaginali cicliche ormono-dipendenti di tipo ematico. I canidi, in realtà, non hanno mestruazioni in senso «umano», ma hanno perdite ematiche che possono essere facilmente scambiate per tali o che, quanto meno, erano certamente scambiate per tali nel mondo antico. Non soltanto: se le mestruazioni delle donne erano considerate le più abbondanti fra i mammiferi, ${ }^{26}$ quelle dei cani, secondo Aristotele, con una durata di circa sette giorni, erano di gran lunga le più prolungate. ${ }^{27}$

Ricordiamo, inoltre, che i canidi non solamente mostrano una particolare attrazione per il sangue (mestruale, ma non soltanto), ma mangiano anche cadaveri, parti del corpo come i genitali, rifiuti di vario tipo ${ }^{28}$ e addirittura escrementi. Ci troviamo di fronte a un peculiare intreccio di un animale «femminile», come il cane, con il mestruo e le feci, ${ }^{29}$ relativamente al quale è con evidenza necessario riflettere.

\section{Consumo di latte e latticini da parte degli Ateniesi}

In questo paragrafo si cercherà di dimostrare che in Grecia, in particolare ad Atene, esistevano pregiudizi per quanto concerne l'uso del latte e dei latticini. Sulla base di essi, soprattutto gli uomini adulti sani non erano soliti

\section{V.n. 32.}

26 Aristotele lo ribadisce a più riprese: GA, I 19, 727a; I 20, 728b; HA, III 19, 520a; VII 2, 582b.

27 Esse coincidono, inoltre, con l'inspessimento degli organi genitali: $H A$, VI 20, 574a (come nel caso di vacche e giumente: VI 18, 572a). Un breve accenno alle «mestruazioni» canine nel mondo antico in Gourevitch 1968, 253. Per le perdite vaginali ematiche negli animali, v. n. 32 .

28 Franco 2003, 111-52.

29 E, nell'opera di Plinio ( $N H$, XXX 43.123), anche con il parto. Plinio, infatti, scrive che la placenta della cagna, messa in contatto con i reni della partoriente, favorisce l'espulsione, a condizione che la placenta non abbia mai toccato il suolo; mentre il latte del medesimo animale favorisce lo sviluppo del feto (ricordiamo che il feto viene nutrito con il sangue mestruale della madre, che successivamente diventerà latte). Sempre secondo Plinio ( $N H$, XXVII 77.250), il latte di scrofa favorisce il parto (e, mischiato con miele, provoca le mestruazioni. In generale il latte di scrofa è molto salutare per le donne: $N H$, XXVIII 77.250). Come ci insegna Semonide nel suo Giambo sulle donne (Fr. 7 West=7 Pellizer-Tedeschi, 1-6), donna e scrofa hanno molto in comune, in particolare per quanto concerne la sporcizia: si può, pertanto, pensare che anche alla base di queste indicazioni terapeutiche ci sia una sorta di principio «simpatico», con particolare riferimento alla farmacopea cosiddetta immonda? Per cane e scrofa, v. n. 34. 
bere latte e guardavano con sospetto ai latticini. Tutto ciò non significa in nessun modo sostenere che gli Ateniesi non consumassero mai latte e latticini o che non li ritenessero (anche) alimenti nutritivi, ${ }^{30}$ significa soltanto segnalare l'esistenza di credenze che potrebbero essere connesse con l'emogenesi del latte animale. A questo scopo si utilizzeranno sia fonti primarie che secondarie.

La riflessione concernente le «mestruazioni» canine ci spinge a fare ulteriori considerazioni circa le «mestruazioni» animali (in particolare quelle degli animali da latte). Per prima cosa, dobbiamo ribadire che le specie animali non presentano vere e proprie mestruazioni, ma soltanto perdite cicliche ematiche, che di solito avvengono durante la fase pro-estrale oppure estrale. ${ }^{31}$ Secondariamente, le uniche specie animali addomesticate dall'uomo che presentano questo tipo di perdite fisiologiche sono i canidi e i bovini. ${ }^{32}$

Questo è quanto ci dice la moderna scienza veterinaria. Per Aristotele, però, le cose stavano diversamente: secondo lo Stagirita, tutti gli animali vivipari, quelli che non producono prima uova (oggi si farebbe la distinzione fra vivipari e ovovivipari), cioè l'uomo e tutti i quadrupedi che hanno gli arti inferiori piegati all'interno, hanno mestruazioni, a meno che non presentino menomazioni congenite (come il mulo). ${ }^{33}$ Bisogna, tuttavia, osservare che

30 Negli stessi testi ippocratici non si lascia molto spazio al dubbio: il latte è alimento nutritivo, il formaggio pure, v.: Vict., II 41, 46 (la carne degli animali che hanno sangue e latte denso è meno digeribile), 51 (Littré VI, 538, 544-46, 554). Cf. infra. È nota anche la polemica presente nel De vetere medicina, 20 (Littrè I, 620-24), nei confronti di coloro che affermano che il formaggio è un cattivo alimento, senza specificare la natura dei disturbi e su quali parti del corpo essa agisca. Gli effetti del formaggio, infatti, risultano molto soggettivi. Un altro passo (Alim., 33, Littré IX 110), però, sembra suggerire qualcosa di diverso: se il latte e il sangue sono ciò che resta del cibo dopo che esso ha nutrito il corpo, si potrebbe dedurre, da un lato, che latte e sangue sono presenti all'interno di ogni nutrimento di origine animale (d'altronde - si sa - i due umori condividono la medesima natura) e, dall'altro, che essi non sono nutritivi.

$31 \mathrm{Nel}$ caso delle vacche, esse avvengono in fase di meta estro ovvero d'estro (cioè subito dopo l'estro). Ricordiamo che, secondo le teorie mediche antiche, il momento migliore per concepire era in prossimità del ciclo mestruale (v. n. 16): questo dettaglio potrebbe aver favorito l'analogia fra mestruazioni femminili e «mestruazioni» animali.

32 Noakes 2008, 20s., 33-35 (stesse pagine per l'edizione inglese). Nelle capre e nelle pecore non si osservano perdite ematiche dalla vagina, ma si verificano perdite di altra entità, come muco, v. Gordon 1997, 55, 375. Perdite vaginali di natura ematica sono state osservate, inoltre, nei mustelidi, v. Stenson 1988, 608. Non sarà forse casuale che anche un animale come la donnola - bestiola dal corpo sottile, domestica nel mondo antico, protagonista di infinite storie, nomi, credenze, che spesso la ricollegano al mondo femminile, in particolare al parto - venga «pensato» come straordinariamente ambiguo, v. Bettini 2008. È necessario sottolineare, però, che le perdite ematiche di un animaletto come la donnola sono di certo poco visibili all'occhio umano.

$33 G A$, I 20, 728b. Cf. $H A$, VII 2, 583a. Cf. anche $H A$, III 20, 521b: tutti gli animali che hanno latte, hanno mammelle; le mammelle sono presenti soltanto negli animali vivipari interni ed esterni (ma non sono presenti nei vivipari soltanto esterni, cioè negli ovovivipari). 
lo Stagirita parla esplicitamente di mestruazioni soltanto per cani, vacche, giumente (i quadrupedi con il flusso più abbondante), capre e pecore. ${ }^{34}$

Ne consegue, sulla base delle teorie antiche, che anche il latte animale utilizzato dall'uomo (in linea teorica tutto il latte animale) è un prodotto visibile del sangue «mestruale».

Vediamo, più nel dettaglio, quale latte era impiegato per uso alimentare e/o medico. Sempre stando alle testimonianze aristoteliche, il latte più delizioso è quello di cammella, da mescolare con acqua ( $H A$, VI 26, 578a); il latte più utilizzato per il formaggio è quello di capra e di pecora, seguito da quello di vacca $(H A$, III 20,522a); il latte più leggero è ancora quello di cammella, seguito da quello di giumenta e quello di asina, mentre il più denso è quello di vacca (HA, III 20 521b). Dal momento che, subito prima, lo Stagirita afferma che il latte più denso è il più ricco di caseina, e dal momento che, poco dopo ( $H A$, III 22, 523a), sostiene che il latte più indicato per i bambini è quello con poca caseina (anche se quello a più alto contenuto di caseina è il più nutriente), ne consegue che il latte vaccino di preferenza non era utilizzato durante l'infanzia. ${ }^{35}$ Altrove si dice che il latte di cagna e quello di scrofa sono i più densi (HA, VI 20, 574b). ${ }^{36}$ Dal Corpus Hippocraticum (Vict., II 41, Littré VI 538), apprendiamo che tutti i ciceoni preparati con il latte sono nutrienti, ma che soltanto il latte di pecora ha proprietà astringenti,

$34 G A$, II 8, 748a; $H A$, VI 18, 572b; VI 21, 575b. La scrofa, come testimonia Aristotele ( $H A$, VI 18, 573a), espelle, dopo l'accoppiamento, una mucosa detta capria. Non si accenna, però, a perdite di sangue. Esopo (223 Perry) racconta di una disputa fra scrofa e cagna sulla fecondità: quest'ultima si vantava di avere, fra tutti gli animali, la gravidanza più breve, ma la seconda le fece notare che i suoi cuccioli nascono ciechi. Per la fecondità della cagna, cf. Antologia greca, IX 268. Cagna e scrofa vengono associate anche in Aristotele (cf.: HA, V 8, 542a; V 14 545b; V 18, 572a) per il fatto di essere animali domestici che, assimilando il comportamento umano, si riproducono più volte l'anno, per il fatto di avere gestazioni brevi e per la precocità dell'accoppiamento. Diversamente da quanto ci si potrebbe aspettare, tuttavia, i termini «cagna» e «scrofa» non venivano utilizzati come insulti indirizzati a donne particolarmente lussuriose: a questo scopo si impiegava, piuttosto, «giumenta» che, come la donna, accoglie l'uomo anche durante la gravidanza. chi lo afferma, e lo ribadisce più volte, è Aristotele, il quale a più riprese sottolinea anche il fatto che le giumente sono gli animali con le perdite mestruali più abbondanti (v. n. 27). Plutarco (18, 111, $290 \mathrm{~A}-\mathrm{C})$ accenna alla lussuria canina, ma prende come paradigma di lascivia la capra (animale da evitare anche per il cattivo odore che emette e per la capacità di trasmettere l'epilessia). Ci si chiede, a conclusione di questa rapida rassegna di fonti, se gli antichi possano aver istituito un nesso fra prolificità, lussuria e perdite vaginali ematiche. Un'analogia fra donne e giumente, proprio in relazione agli effetti del sangue mestruale, si trova in Plinio, v. n. 11.

35 Probabilmente veniva utilizzato il latte di capra e di pecora che, al di là di tutte le articolate riflessioni del filosofo sui vari tipi di latte, era il più utilizzato nel mondo antico, v.: Montanari 2000, 12-14; Cicala 2000, 37. Cf. n. 36.

36 Vediamo anche l'opinione «romana»: secondo Plinio ( $N H$, XXVIII 33.123. L'intero paragrafo è dedicato all'uso del latte), il latte di capra è il più nutriente (dopo quello materno, che risulta anche essere dolce, delicato e buono: $N H$, XXVII 21.72) e quello più indicato per lo stomaco, quello di asina il più attivo (un'emina veniva somministrata ai bambini prima dei pasti. In alternativa, una di capra: $N H$, XXVIII 33.126), quello di vacca il più curativo e 
il latte di capra rilassanti, il latte di vacca meno, il latte di cavalla e quello d'asina di più.

Latte e i latticini, dunque, sembrano presenti nella dieta ateniese. ${ }^{37}$ Aristotele non specifica chi fossero i consumatori di questi prodotti, ma possediamo numerose testimonianze che ci portano a pensare che il cittadino maschio di Atene di norma evitasse il consumo del latte (e forse, possibilmente, anche di alcuni suoi derivati), a causa di un pregiudizio assai radicato che, in maniera più o meno conscia, sarebbe potuto derivare non soltanto dalla natura ambigua e instabile del latte,${ }^{38} \mathrm{ma}$ anche dalle teorie relative alla sua emogenesi.

il più lassativo, quello di cammella il più dolce. In generale, il latte di animali e di donne di grossa taglia, secondo Plinio, è più digeribile. Di opinione diametralmente opposta Aristotele (HA, VII 12,588a), secondo il quale il latte troppo grasso e proveniente da nutrici troppo corpulente può causare convulsioni al bambino.

37 Per quanto concerne i derivati del latte (formaggi, burro, «yogurt») sono conosciuti e consumati, anche se, a esempio, nelle ricette si preferisce l'olio di oliva al burro (che si deteriora in tempi più brevi ed è più difficile da ottenere). Il burro, però, è utilizzato al posto dell'olio dagli abitanti delle montagne (öpєıo: Strab., III 3.7). Cf.: Dalby 1996, 66; Dalby 2003, 65, 80 s., $217 \mathrm{~s}$.

38 Secondo Plinio (XXVIII 45.158), il latte di asina è un antidoto contro i veleni e contro il latte cagliato dentro lo stomaco, dal momento che questa coagulazione (coagulum) del latte agisce come un veleno. Alimento e medicamento allo stesso tempo, il latte può essere contemporaneamente veleno, antidoto e veicolo di malattie. Con le sue ambigue caratteristiche, rientra a pieno titolo nella categoria dei фápнaкa con effetti contraddittori, in quanto per sua stessa natura è formato da elementi contrari (Orib., Collect. Med., II 59). Gli antichi sono stati enormemente affascinati dal processo di accagliatura del latte, basato sulla «antipatia» fra i componenti, quindi sulla separazione. A partire da Aristotele (GA, II 4, 739b: пveTía;

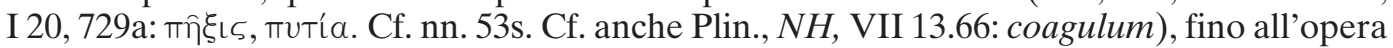
di sant'Alberto il Grande (1206-1280), l'immagine del latte cagliato è stata utilizzata per spiegare il processo del concepimento. La funzione essenziale di questa metafora è quella di rappresentare il passaggio dalla stato liquido (del seme e del sangue) allo stato solido (del corpo del bambino). L'accagliatura è l'esatto opposto rispetto alla burrificazione: quest'ultima è lenta e l'uomo ne è l'artefice; la prima, invece, è rapida, quasi immediata, ai limiti del «miracoloso». È interessante notare che il verbo «far cagliare» in greco si traduce con $\uparrow \rho \epsilon ́ \phi \omega$ (cf. n. 71). Come osserva Demont 1978, 263, esso rappresenta un «universel de langage où sont associées croissance, coagulation, solidification et transformation». Crescita e coagulazione non sono concetti antinomici nella cultura greca. Allo stesso modo, non vi è contraddizione fra «far prendere», «prendere corpo» e «nutrire»: il feto «prende corpo» nutrito dal sangue materno, «prende forma» diventando un prodotto elaborato come il formaggio. Non a caso, l'italiano «formaggio» deriva dal latino forma. La gravidanza non soltanto permette la crescita del corpo del bambino, ma anche la sua solidificazione (ed eventualmente la sua deformazione, essendo il feto materia ancora molle e malleabile). Questa metafora ha, inoltre, contribuito a rafforzare l'idea, tanto chiara - e cara - ad Aristotele, che il seme maschile potesse agire come principio immateriale sulla materia femminile, nello stesso modo in cui la coagulazione nel processo di accagliatura non avviene «materialmente», ma in virtù del calore che fa cagliare il latte (cf. GA, I 22, 730b). Superfluo ricordare per l'ennesima volta che il latte era considerato il sangue mestruale, che costituiva, prima, la suddetta «materia» (Nat. puer., 14, Littré 492), e con cui, dopo, veniva nutrito il feto. Per ulteriori remarques su quella che, con Jackie Pigeaud, potremmo definire la «rêverie de la caille du lait» nella cultura greca, si veda, appunto, Pigeaud 1975, in particolare 15-17. Si veda anche Bodiou 2011, 148s. Forse non è casuale il fatto che Circe aggiunga i фáppaka che causeranno 
Il latte animale è ampiamente attestato, senza specificazioni di genere per quanto riguarda i suoi destinatari, nella farmacopea ippocratica: ${ }^{39}$ questo dato ci informa inequivocabilmente circa la sua natura di liquido «particolare». Il latte umano, invece, risulta utilizzato, in maniera a nostro avviso non troppo sorprendente, soltanto per donne, e in rari casi per bambini. ${ }^{40}$

ai compagni di Odisseo l'oblio della terra paterna, trasformandoli in porci, proprio a un intruglio (definito da Odisseo ciceone) di formaggio ( Tupóv), farina d'orzo, miele e vino (Hom., Od., X 234-36, 316). E forse non è un caso che siano sempre donne, in particolare donne legate alla sfera della magia, a preparare questo tipo di intrugli (v. Albertocchi 2012, 71). Stando ad alcune descrizioni degli ingredienti, il latte era contenuto nel ciceone preparato in occasione dei misteri eleusini (ma non è presente nella ricetta di Hom., 2, 209, in cui si sottolinea l'assenza di vino nella bevanda preparata per la dea, non si parla di latte, però si menziona, come abbiamo visto, il formaggio). Cf. n. 57. Secondo Dalby 2003, 190, кUкєúv deriva dal verbo кикáw, che veniva utilizzato, fra le varie accezioni, anche per indicare l'azione di colui che mescola il latte. Per la preparazione del ciceone, v. Delatte 1954, 71027. Il ciceone è preparato anche a scopo curativo, v. supra.

39 Si vedano, a titolo di esempio: Epid., VII 3s. (Littré V 368-72: siero, latte di asina, di vacca, di capra, in tutti i casi per dolori al ventre), VII 115 (Littré V 462: latte di capra, per rilassare il ventre); Aph., V 64 (Littré IV 556s.: latte in generale, per i tisici, per coloro che soffrono di febbre non alta ma di lunga durata, in casi di deperimento estremo); Morb., II 51 (Littré VII 80: siero o latte di asina, e poi di vacca, in caso di tubercolosi vertebrale, dopo una purga); Int., 16 (Littré VII 206: siero o latte, per rilassare il ventre). Ci sono, inoltre, testimonianze più tarde, a esempio due iscrizioni rinvenute all'interno di santuari di Asclepio. In entrambi i casi il malato che si rivolge al dio soffre di problemi di stomaco: Prêtre/Charlier 2009, 151 (Lebena, II-I secolo a.C.: latte, senza alcuna specificazione), 191 (Epidauro, 160 ca. d.C.: latte con miele. Chi scrive specifica che prima era solito, sbagliando, berlo puro, $\mu$ óvov). Per altre patologie, però, è vivamente sconsigliato, come si legge nel medesimo aforisma: Aph., V 64 (Littré IV 556s.: per esempio in caso di cefalgia o di febbre alta). Si noti che si tende a preferire il latte diluito con acqua, detto idrogala. Cf. Auberger 2010, 103-110; Deichgräber 1971 (in particolare per Ep., VII). Segnaliamo un dettaglio assai singolare: in Mul., I 43 (Littré VIII 100-102) si sottolinea che il latte utilizzato nel caso in cui la donna vomiti sangue dopo il parto deve essere quello di una vacca nera, preso a digiuno (dopo quello di asina). Auberger 2010, 106, ipotizza che il latte di vacca nera sia più ricco, in base alla stessa logica per cui il latte di una donna bruna è più ricco di quello di una donna bionda.

40 Laskaris 2008. L'impiego principale era nella preparazione di pessari vaginali. Il latte di

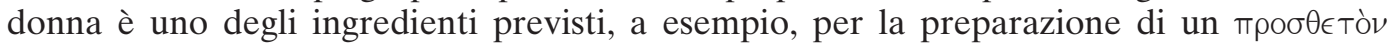

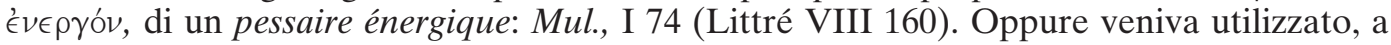
esempio, in caso di leucorrea, per la quale si consigliava di bere siero, latte di vacca e/o latte diluito: Mul., II 118s. (Littré VIII 254-58); Nat. mul., 15 (Littré VIII 332-34). Ricordiamo che la leucorrea si manifesta - forse non a caso - con perdite vaginali, più o meno vischiose, di color biancastro-trasparente; e notiamo altresì che il latte utilizzato è quello vaccino. La Laskaris 2008, 460, evidenzia che il latte umano era effettivamente uno dei più potenti antibatterici conosciuti nel mondo antico. A Roma la situazione appare significativamente diversa. Da un lato, secondo Bonnet 1995, 164, nella farmacopea pliniana il latte, bevanda dalle proprietà particolarmente addolcenti ed emollienti e in generale molto utilizzata, risulta avere maggiore efficacia se somministrato a donne incinte e a bambini; dall'altro, però, non soltanto il latte animale, ma anche quello umano risultano assai impiegati, apparentemente senza distinzioni di sesso, nelle ricette riportate da Plinio: NH, XXVIII 21.72-74, 47.171, 48.173, 74.241; XXX 22.72, 39.118. L'indicazione più singolare e degna di nota senza proviene ombra di dubbio da Galeno (VII $701 \mathrm{~K}$ ): il latte umano veniva utilizzato per curare patologie senili e risultava ancora più efficace se bevuto direttamente dal seno muliebre. Leggendola viene inevitabilmente da commentare che i vecchi sono due volte bambini da molteplici punti di vista! Non si può non pensare, a questo proposito, al caso di Pero e 


\section{A ciò aggiungiamo le testimonianze relative a popoli barbari mungitori di cavalle e/o mangiatori di latte ${ }^{41}$ e bevitori di latte ${ }^{42}$ (e come non pensare, poi, al caso di Polifemo?). ${ }^{43}$}

Micone, ma l'allattamento filiale, cui è stato dedicato un Colloquio (Raffaelli/Danese/ Lanciotti 1997; cui segue Danese 2000 sulla fortuna del tema nei secoli), meriterebbe un discorso a parte. Ancora nel XIX secolo il latte femminile era considerato particolarmente efficace a fine terapeutici (per esempio per curare l'emottisi e la tisi), soprattutto se attinto direttamente alla «fonte», v. Camporesi 1993, 13s.

41 Hom., Il., XIII 5 (cf.: Strab., VII 3.9, 4.6); Callim., H., 3, 252 (in questo caso i Cimmeri sono

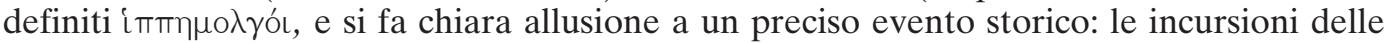
popolazione nomadi del Bosforo, che attaccarono Sardi e si spinsero fino in Cilicia fra VIII e VII secolo a.C. Difficile pertanto pensare a una valenza anche soltanto parzialmente positiva del termine in un simile contesto). Barbari, però, meno «barbari» degli altri: Hom., Il.,

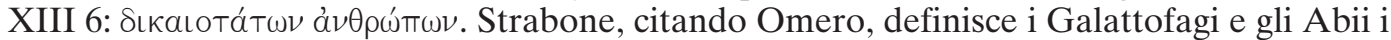
più giusti fra gli uomini. Cf.: Strab., VII 3.9; 70F42 Jacoby = 151 M.-W. (in cui si menziona

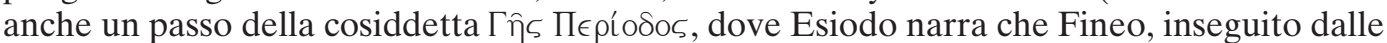
Arpie, giunge al paese dei Galattofagi, che utilizzano i carri come dimore). V. Piasere 2008.

42 Hdt., I 216.4 (Massageti); III 23.1 (Etiopi); IV 186.1 (Libici). Cf.: Eur., El., 169: «è arrivato un uomo che beve latte ( үа入актото́таs), un miceneo che vaga per i monti» (trad. S. Fabbri, Milano 1995. Cf. n. 48). Per il mondo romano, v.: Plin., NH, XI 96.239 (barbarae gentes, quae lacte vivunt); Tac., Germ., 23; Caes., Bell. Gall., V 14, VI 22. In entrambi i passi Cesare sta parlando dei Britanni, di cui sottolinea con insistenza le abitudini alimentari a base di carne e latte e il «comunismo» delle donne e della proprietà. Notiamo, nondimeno, l'aggettivo in grado superlativo humanissimi. Anche Plinio e Tacito non esprimono un giudizio negativo

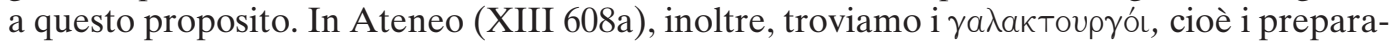
tori di formaggi (cf. Poll., I 251) ovvero i cuochi specializzati in piatti a base di latticini: siamo alla corte persiana. Fonti medievali relativamente alle popolazioni consumatrici di latte anziché di vino in Montanari 2000, 11s. «La barbarie»- si domanda retoricamente Montanari - «non è forse una forma di infanzia culturale?»: Montanari 2000, 9-12; Montanari 2001, 9s. Cf. Auberger 2010, 97-99. Montanari osserva, inoltre, che nei testi medievali vi sono riferimenti al consumo di latte da parte degli adulti soltanto - e non a caso - nei periodi di carestia. È interessante notare, a questo proposito, che normalmente le donne in Grecia non dovevano bere vino (cf. n. 57), ma che, stando invece all'opinione di Sor., Gynaec., II 26, era concesso loro berlo durante l'allattamento per curare l'ipogalattia. Ovviamente la nutrice deve astenersi dall'ebbrezza, v. Sor., Gynaec., II 19. Dettaglio ancora più singolare: l'alimentazione della nutrice, sempre secondo Sorano, apparentemente non prevede latte: forse per evitare l'intromissione di un altro tipo di latte nel suo latte?

43 Hom., Od., IX 187-92: Polifemo viene dipinto come un mostro gigante che in disparte pasce le greggi. Di lui Odisseo subito afferma che non ha sembiante di «mangiatore di pane»,

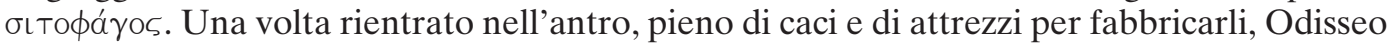
lo descrive intento ad operazioni di mungitura di pecore belanti. Ma sono le parole con cui Odisseo dipinge il gigante sazio della carne dei compagni che maggiormente colpiscono: «quando il Ciclope ebbe riempito il gran ventre, carne umana mangiando e latte puro bevendo, si distese nell'antro, sdraiato in mezzo alle pecore» (Hom., Od., IX 296-98. Trad.

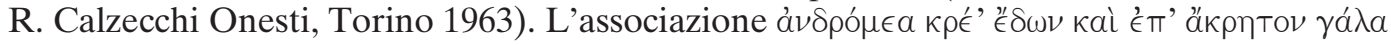
Tí $\nu \omega \nu$ è particolarmente pregnante, così come l'immagine del gigante che, dopo l'empio

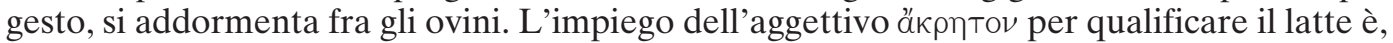
inoltre, interessante: nella cultura greca è il vino che si beve äkрaтos, «puro». Il pastore Polifemo è, in breve, «un galaktophagos che non resiste alla carne fresca e al vino puro»: Hartog 2002, 31. 
L'insieme di queste testimonianze confermano e arricchiscono la riflessione relativa alla natura del latte come bevanda dalle straordinarie implicazioniculturali. Cerchiamo, allora, di approfondirne ulteriormente il significato come alimento.

Pur riconoscendo che il formaggio è forte, molto riscaldante e nutriente $\mathrm{e}$ che il latte ha alto potere nutritivo ${ }^{44}$ sostanzialmente esso viene consigliato soltanto per uso medicinale, sottolineando i numerosi pericoli del latte sotto il profilo alimentare. ${ }^{45}$ L'associazione del formaggio all'idea di impurità è evidente in un passo di Artemidoro (I 72), in cui si afferma che sognare di mangiare focacce senza formaggio è buon segno, mentre sognare di mangiare focacce con formaggio è interpretato come segno di inganno e insidie, poiché tale è il significato del formaggio. Sempre Artemidoro (I 16) afferma che gli adulti si nutrono di latte soltanto quando sono malati e non possono assumere cibo in forma solida.

La profonda connessione culturale - oltre che fattuale e, per così dire, «tecnica» - tra latte e infanzia ha verosimilmente contribuito all'impossibilità di considerare il latte $\mathrm{e} i$ suoi derivati come cibo con un valore alimentare - e culturale - totalmente positivo (accanto, ovviamente, a ragioni di carattere ambientale e climatico). Come alimento per l'età adulta sembra quasi rifiutato, mettendo in luce un atteggiamento di diffidenza, forse in qualche caso anche di disprezzo, verso qualsiasi tipo di latte che venga proposto al di fuori del contesto nutrizionale infantile. ${ }^{46}$ Considerando, oltre a ciò,

44 Plut., 11, 19132B, indica il latte non come bevanda, ma come cibo (cf. Varr., Re rust., II 11.1). Auberger 2010, 105, deduce che il latte non era considerato una bevanda in Grecia anche dall'assenza di esso nella lista delle bevande in Vict., II 52 (Littré VI 554-556) e da un epigramma anonimo dell'Antologia greca (XI 297), in cui una madre suggerisce al figlio, che chiede alla donna, che un tempo gli ha dato del latte, del vino, ma la donna gli risponde di bere acqua per dissetarsi (in merito all'interpretazione di quest'ultima testimonianza ci pare, però, si possano esprimere perplessità. Si tratterà, piuttosto, di una fonte che attesta il costume di allattare, ma in un'epoca molto più tarda). Stando, inoltre, alla testimonianza di Diogene Laerzio (VIII 12), fu un certo Pitagora (probabilmente non il celebre filosofo di Samo) a indicare per gli atleti una dieta a base di carne, a posto di quella a base di fichi e formaggi. Troviamo, dunque, il formaggio all'interno di un'alimentazione eccezionale. Come è noto, nell'opinione dei medici antichi le diete hanno destinatari ben precisi e una dieta non adatta al soggetto per cui è stata pensata nuoce gravemente alla salute. Si tratta, però, di fonti assai tarde.

45 Aph., V 64 (Littré IV 556s.). Anche Gal., XVII/2 872-77 K. Cf. Naso 1990, 67.

$46 \mathrm{E}$, forse, femminile. Ancora oggi nel Nord Europa (dove il consumo di latticini è storicamente assai radicato, v. Camporesi 1993, 21-25) esistono numerosi rituali dedicati a divinità femminili in cui il latte e i suoi derivati hanno un ruolo centrale, v. Davidson 1996. È singolare, oltre a ciò, il nesso esistente in alcune culture fra malocchio e latte (e derivati del latte), che porta Djéribi 1988, 41, a concludere che: «le lait apparaît comme un liquide éminemment conducteur du mauvais æeil». Cf. n. 22. Ricordiamo, inoltre, che, secondo Aristotele (GA, III 784a. Cf. GA, I 20,728a), la natura delle donne è più simile a quella dei bambini che non a quella degli uomini. 
l'ampio impiego di latte a fini terapeutici, ${ }^{47}$ possiamo forse concludere che il greco maschio adulto, che viveva nella polis e che poteva permetterselo, tendenzialmente non beveva latte (liquido nient'affatto scevro di radicate valenze culturali), se non quando era malato. ${ }^{48} \mathrm{Il}$ fatto di essere sangue mestruale cotto può aver contribuito, come abbiamo ipotizzato, ad alimentare (e giustificare) un atteggiamento di sospetto e di rifiuto nel confronti di questa potente bevanda «naturale». ${ }^{49}$

Non dimentichiamo, a questo proposito, l'opinione di Empedocle, secondo il quale il latte sarebbe addirittura sangue putrefatto. Come riporta lo stesso Aristotele con parole critiche ( $G A$, IV 8, 777a), secondo Empedocle

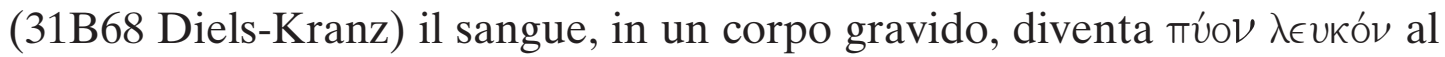
decimo giorno dell'ottavo mese. Il passo aristotelico, da cui è tradito il frammento, chiarisce che si sta parlando delle relazioni fra sangue, latte e seme. Come è stato osservato, ${ }^{50}$ il frammento medico di Empedocle conterrebbe

47 V. nn. 39s. Segnaliamo una scoperta che ci sembra interessante: in un villaggio romano nei pressi di Cartagine sono stati rinvenuti due recipienti, identificati come biberon, provenienti da una sepoltura e datati II secolo d.C. (uno contenente tracce di lattosio, uno privo di tracce al suo interno). Secondo Quevedo Sánchez 2010, essi potevano essere utilizzati per l'alimentazione di persone malate, non in grado di ingerire cibo solido (ma non si escludono altri usi): questa interpretazione ci pare particolarmente significativa, soprattutto se messa in relazione alle fonti che testimoniano l'assunzione di latte o di suoi derivati in caso di malattia.

48 Verosimilmente diversa la situazione fuori da Atene, in particolare nelle zone rurali. Cf.:

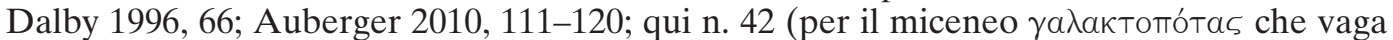
per i monti). L'argomento in realtà è assai controverso e non è facile esprimersi in merito. Qui si vuole mettere in evidenza una tendenza, una «preferenza» alimentare, un pregiudizio. I formaggi non mancavano di certo nell'alimentazione ateniese: erano, a esempio, un prodotto molto presente nei mercati, anche se i venditori di formaggio erano spesso oggetto di scherno e di offesa nella commedia e di questo alimento si sottolinea il basso costo. Per Platone ( $R p$., II 372c) fa parte degli alimenti sani con cui nutrire i cittadini, secondo un'alimentazione tipica delle campagne. Un dettaglio ci pare significativo: la «scoperta» del formaggio nel mito è attribuita alle ninfe, le quali avrebbero insegnato ad Aristeo, figlio di Apollo, l'arte di cagliare e trasformare il latte (Diod., IV 81.2. Cf. Oppian., Cyneg., IV 265 76). Si noterà con facilità che sia le «inventrici» del formaggio, sia il più noto «produttore» e consumatore di esso nel mito e nell'epopea sono personaggi ambigui, marginali, selvatici, per molti aspetti «altri». Diodoro, però, sia per l'epoca in cui scrive sia per la sua provenienza, è una fonte da utilizzare con cautela.

49 La mancanza di latte e latticini nell'alimentazione dell'adulto può avere molteplici cause: intolleranza al lattosio, motivi squisitamente culturali (soprattutto nell'Oriente asiatico), ragioni climatiche e ambientali (accanto al problema della conservazione del latte, nell'Europa meridionale si coltivano - e si coltivavano - principalmente vite e ulivi). V.: il Capitolo Lattofili e lattofobi in Harris 1990; Davidson 1996, 91s. Ma esiste anche un diverso «milk complex», spiegato da Matthey 1989 a proposito dei Bantu orientali, «i quali attribuivano al latte il valore supremo, non tanto come unico alimento, anche se cibo preferito, quanto come il bene che riassume in sé ciò che è positivo e desiderato esiste sulla faccia della terra. La sua mancanza equivarrebbe a un disastro e alla fine di quel mondo, che lo ha innalzato a proprio emblema e simbolo. La sua preminenza è di carattere culturale. Ne consegue che esso è considerato superiore ai cibi solidi, cui si trova deliberatamente contrapposto.»

50 King 1987, 121-23; Caratozzolo 2007, 138. 
un gioco di parole fra túov (pus, intendendo il latte) e пuós (il primo latte dopo la nascita, cioè il colostro). Dalle scarne informazioni in nostro possesso, secondo Empedocle il latte è sangue che ha subito un processo di decomposizione; mentre per Aristotele il latte ha subito il processo esattamente contrario, ovvero la cottura (cioè è cotto, ma non corrotto ${ }^{51}$ ). Aristotele, come abbiamo detto, critica la teoria empedoclea, ma non esita a definire

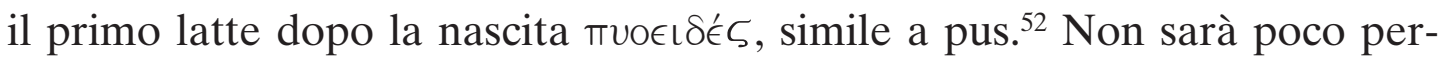
tinente ricordare, allora, che il processo di accagliatura del latte, che viene spesso paragonato al processo di coagulazione del sangue ${ }^{53}$ e che viene sovente utilizzato metaforicamente per descrivere la formazione dell'individuo a partire dal sangue mestruale materno e dallo sperma paterno, ${ }^{54}$ è di

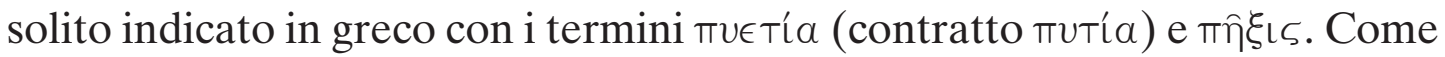

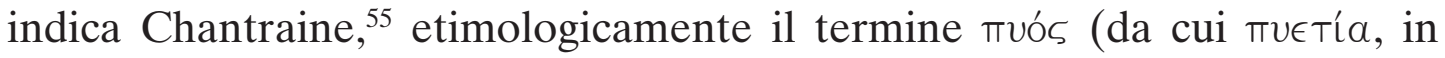
greco moderno Tuтía) va messo probabilmente in relazione con múov, dal momento che «les notions de se coaguler, surir, fermenter, peuvent s'associer à celle de se corrompre».

Consideriamo, per concludere, una problematica testimonianza tratta dal Corpus Hippocraticum: Alim., 33 (Littré IX 110). Il passo, piuttosto ellittico e isolato rispetto ai paragrafi precedenti e successivi (soltanto nel paragrafo successivo a quello in questione si accenna al fatto che esistono diversi tipi di alimentazione: un'alimentazione per bambini, che ha lo scopo di nutrirli e di farli crescere; un'alimentazione per vecchi, che ha soltanto lo scopo di nutrirli; un'alimentazione per atleti, che ha lo scopo di farli diventare più forti) è di non facile interpretazione. Littré lo intitola: «Convenances des diverses espèces d'aliments suivant les diverses conditions» e ne offre la seguente traduzione: «lait, nourriture pour celui à qui le lait est nourriture, conformément à la nature, à d'autres non; vin, nourriture à ceux-ci, et non à ceux-là, ainsi que la viande et beaucoup d'autres espèces de nourriture, suivant le pays et suivant l'habitude». ${ }^{56}$ Dalla traduzione dello studioso francese si potrebbe intuire che il latte è un nutrimento adatto soltanto a coloro per $\mathrm{i}$ quali è prodotto, cioè ai poppanti. Il riferimento al vino, bevanda notoria-

51 V.n. 5.

$52 H A$, VI 18, 573a. Il latte secondo lo Stagirita assomiglia a pus, ma soltanto all'inizio della gestazione.

53 Hom., Il., V 902-04; Aeschl., Ch., 66s. Cf. Pigeaud 1975.

54 V.n. 38.

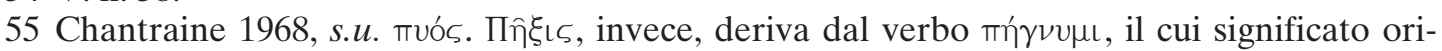
ginario di «fixer, d'où construire, être solide, fixe, a conduit à des emplois très divers [...]

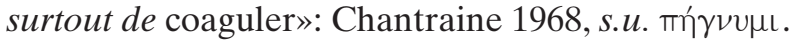

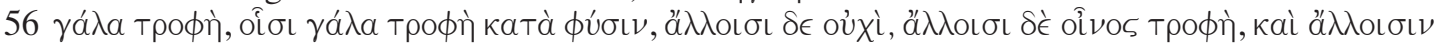

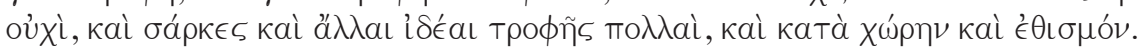


mente non indicata per tutti, sembra andare in questa direzione, ${ }^{57}$ così come in greco кaTà $\phi \dot{\sigma} \sigma \nu$ sembrerebbe confermare quest'ipotesi, che, però, va considerata soltanto come tale, data la mancanza di informazioni nel testo. La domanda che Clemente Alessandrino retoricamente pone circa la ragione per cui non veniva più utilizzata la prima fonte di alimentazione del bambino, ovvero il latte, cui le nutrici abituano sin dalla nascita, ${ }^{58}$ sembrerebbe tuttavia accreditare, in un'epoca evidentemente molto diversa, quella che abbiamo appena definito soltanto una mera ipotesi.

\section{I diversi tipi di latte e la trasmissione di caratteri ereditari attraverso il latte umano nei testi medici}

In questo paragrafo si vuole dimostrare l'esistenza in filigrana di diversi tipi di latte anche nei testi ippocratici. Questa differenza, da cui deriva la necessità di grande attenzione nella scelta della nutrice, era posta particolarmente in evidenza dai medici a Roma, ma non era assente in Grecia, dove, però, essendo il baliatico molto meno frequente, soprattutto in epoca arcaica e classica, emergeva con minore insistenza. Si utilizzeranno principalmente fonti primarie, per quanto di difficile interpretazione.

Mentre i medici di epoca imperiale hanno scritto fiumi di parole sulla pratica dell'allattamento, soprattutto in relazione al costume, assai diffuso all'epoca, di non allattare personalmente i propri figli ricorrendo a balie, le fonti greche parlano pochissimo dell'argomento in questi termini. La domanda che si siamo posti di fronte a questa mancanza, che verosimilmente non va attribuita solamente al cosiddetto «naufragio delle fonti», è la seguente: tutte le problematiche, che stavano particolarmente a cuore ai Romani, relative alla natura del latte umano e gravitanti intorno alla possibilità di contaminare il sangue della gens attraverso l'impiego di nutrici, erano estranee alla mentalità greca? Oppure non vengono sollevate con la medesima frequenza soltanto perché, non essendo usuale il costume di utilizzare balie per l'allattamento, il problema in questi termini non si poneva? Stando alle fonti in nostro possesso, infatti, le madri greche, perlomeno in epoca arcaica e clas-

57 Che il vino sia bevanda per eccellenza maschile è risaputo, ma la Albertocchi 2012 mette in evidenza il fatto che anche le donne, in alcune precise circostanze, potevano consumare bevande inebrianti, a esempio l'idromele: il miele, come il latte, era infatti una sostanza collegata alla sfera muliebre ed infantile per il carattere «liminale». Cf. nn. 42, 46.

58 Protr., X 1. Di certo Clemente conosceva (e condivideva) la teoria dell'emogenesi del latte, v. n. 9. Cf. Davidson 1996, 92. 
sica, tendenzialmente allattavano i propri figli. Ne abbiamo esempi importanti nell'epopea e nella tragedia, ma non soltanto. ${ }^{59}$

Tornando all'impiego di latte umano nelle terapie mediche, risulta particolarmente rilevante la distinzione, presente già nei testi egizi, fra latte prodotto per una figlia femmina e latte prodotto per un figlio maschio. A questo proposito, i medici dei trattati ippocratici non ne parlano tanto per le presunte proprietà terapeutiche, quanto piuttosto per alcune singolari capacità che rivelano il carattere «sessuato» del latte umano. Una delle più curiose funzioni che vengono ad esso attribuite è, infatti, quella di predire il sesso del nascituro. In Ster., 216 (Littré VIII 416), leggiamo: «prendre du lait de la femme, le pétrir avec de la farine, former un petit pain et le faire cuire sur un feu doux; s'il se calcine, elle est grosse d'un garçon; s'il s'entr'ouvre, d'une fille. Recevoir ce même lait sur des feuilles et l'exposer eu feu; s'il se coagule, c'est un garçon; s'il entre en déliquescence, c'est une fille. $\gg^{60}$ In Ster., 214 (Littré VIII 414), invece troviamo, fra i vari e bizzarri metodi per sapere se una donna riuscirà a concepire, la seguente indicazione: «donnez à boire le matin à jeun du beurre et du lait de femme nourrissant un garçon; si la femme a des éructations, elle concevra; sinon, non.»

In quest'ultimo passo ritroviamo la distinzione fra latte prodotto per una femmina e latte prodotto per un maschio, che vorremmo ulteriormente approfondire. Secondo Plinio, ${ }^{61}$ uno dei fattori più caratterizzanti del latte è il sesso del bambino per cui esso è stato prodotto: come, secondo Aristotele, c'è un seme maschile attivo e uno inerte; così, secondo Plinio, c'è un latte «maschile» più efficace e un latte «femminile» meno efficace. Se esistesse un rapporto fra le tesi aristoteliche e le notizie riportate da Plinio - e alcuni det-

59 Ecuba: Hom., Il., XXII 83; XXIV 58; Andromaca: Eur., Tr., 757; Andr., 224s. (cf. infra); Giocasta: Eur., Ph., 987, 1527 (cf. infra). Soltanto per citare gli esempi più noti. V.: Molinos Tejada 2001; Molinos Tejada 2005.

60 Come nota la Bodiou 2006, 163, quest'esempio sottintende un'analogia fra fare il pane e fare un bambino, la qual cosa implicherebbe l'idea dell'utero femminile come un forno, cf. n. 9.

61 In XXVIII 21.72, si afferma che, per ogni uso, è più efficace il latte di una donna che ha messo al mondo un figlio maschio e di gran lunga più efficace è quello di colei che ha generato due gemelli maschi. Il latte di una donna che aveva partorito un maschio veniva utilizzato soprattutto per problemi agli occhi (XXXVI 20.145), ma anche, come abbiamo visto, per evitare che i cani contraessero la rabbia (v. n. 22); mentre quello di una donna che aveva generato una femmina era preferibile soltanto per curare le affezioni cutanee del viso (XXVIII 21.75). L'uso del latte di una donna che ha partorito un maschio, mischiato con miele, per guarire malattie degli occhi è attestato anche in un testo buddhista appartenente ai Mahāyāna, tradotto in cinese a partire da un originale sanscrito scritto prima del 650 d.C. In esso si specifica che il latte di una donna che ha partorito una femmina non ha alcun effetto. V. Triplett 2010, 490. Il fatto di ritrovare la medesima indicazione in testi prodotti da culture così diverse (Egitto, Grecia, Roma e India), ma non così distanti, ci sembra un dato di straordinario interesse. Con ogni evidenza alla base di credenze di questo tipo deve esserci l'idea che il latte sia in grado, come il sangue, di veicolare caratteri genetici. 
tagli presenti nel Corpus Hippocraticum -, si potrebbe allora dire che il latte «maschile» è più valido perché deriva dal sangue che, al momento del concepimento, ha ricevuto dal seme paterno quella maggiore quantità di calore e quell'impulso più forte capaci di provocare la formazione di un individuo di sesso maschile. Quindi il latte, come il sangue che lega i membri della stirpe, porta in sé e trasmette, secondo i vari usi che se ne fanno, l'intensità dell'impronta formatrice del genitore quale si rivela nei caratteri acquisiti dal figlio, traducendosi anche in potenziale forza fecondatrice. In effetti è probabile che gli antichi ritenessero che la «virilità» impressa dal seme paterno nella materia femminile, in modo da generare un figlio maschio, continuasse ad essere presente e attiva anche nel sangue destinato a trasformarsi in latte e, quindi, nel latte stesso. In questo modo si spiegherebbe la curiosa pratica indicata nel De sterilibus: il latte maschile mantiene, in un certo modo, una continuità con il seme da cui è stato generato, riuscendo ad avere una capacità di testare la fertilità femminile: per gli antichi la fecondazione comportava una sorta di «cottura» 0 «digestione $»^{62}$ della materia fertile femminile da parte del seme maschile; se allora la donna, ingerendo del latte «maschile», riusciva a digerire, come prova il suo rutto, ciò garantiva la sua positiva reattività al calore, quindi la sua fecondità.

Veniamo alla scelta della nutrice. Plinio, Galeno, Oribasio, Mnesiteo: tutti si sono preoccupati di aiutare nella scelta della giusta nutrice (che doveva somigliare sia fisicamente che moralmente il più possibile alla famiglia del bambino) e di istruire la nutrice circa alcuni comportamenti da mantenere (come, a esempio, non rimanere di nuovo incinta durante l'allattamento). ${ }^{63}$ Sorano dedica addirittura un'intera parte della sua opera (Gyneac., II 19) a questo problema.

62 Il verbo greco $\pi \epsilon ́ \sigma \sigma \omega$ con cui Aristotele indica di solito la cozione significa sia «cuocere» che «digerire».

63 Le nutrici greche a Roma erano particolarmente apprezzate per la loro lingua, che il piccolo aristocratico romano, futuro bilingue, avrebbe iniziato ad apprendere già al seno della balia. La nutrice, però, può trasmettere un bagaglio culturale talvolta giudicato sospetto, poiché trasmette credenze che possono sfuggire al controllo cdei genitori, come nel caso fabulis nutricularum, v. Dasen 2010. Cf. n. 20 sulla «levatrice-strega». La tendenza dei moralisti «pagani» prima, dei cristiani poi, a incoraggiare l'allattamento materno è forse stata rafforzata, in un'epoca in cui il Cristianesimo si impone come religione dell'Impero, dalle perplessità di fronte al rischio di assumere nutrici poco istruite sul nuovo credo, perplessità di certo presenti nella società bizantina, v. Rey 2004. Anche nel mondo arabo si ritiene che il latte derivi dall'apporto spermatico dell'uomo che ha fecondato la nutrice, per cui si ha una forte considerazione, soprattutto per quanto riguarda le proibizioni matrimoniali, anche della fratellanze di latte, v.: Khatib-Chahidi 1992; Parkes 2005. Ma l'idea che il latte umano sia in grado di trasmettere non soltanto caratteri genetici, ma anche il carattere e un bagaglio di credenze di tipo etico e di conoscenze culturali, è diffusa presso numerosi popoli non soltanto del passato, v. Maher 1992a. Fra i numerosi esempi non concernenti il mondo antico in cui ritroviamo la teoria dell'emogenesi di latte e sperma e la conseguente idea che anche 
Questo a Roma. Invano cercheremmo qualcosa del genere in Grecia, dove il problema della scelta della nutrice non era apparentemente così sentito. ${ }^{64}$ Abbiamo, però, alcune informazioni «sparse» qua e là.

Già in Aristotele e nei testi ippocratici, come abbiamo visto, si può leggere fra le righe la distinzione fra un latte «maschile» e uno «femminile», con tutte le implicazione che una simile diversificazione comporta. Mnesiteo, affermando che il latte aveva peculiarità tali da renderlo adatto soltanto all'individuo dal cui sangue era derivato e per il quale era prodotto, conferma perlomeno nel IV secolo a.C. - l'esistenza di simili credenze.

Aggiungiamo un'altra fonte: Alim., 40 (Littré IX 112). Leggiamo la traduzione di Littré: «sang d'autrui, utile; sang propre, utile; sang d'autrui, nuisible; sang propre, nuisible; humeurs propres, nuisibles; humeurs d'autrui, nuisibles; humeurs d'autrui, favorables; humeurs propres, favorables; le concordant, discordant; le discordant, concordant; lait d'autrui, bienvenu; lait propre, nuisible; lait d'autrui, nuisible; lait propre, utile». ${ }^{65}$

Troviamo in questo passo l'indicazione dell'esistenza di un sangue alieno utile, di un sangue proprio inutile, di una sangue alieno nocivo, di un sangue proprio nocivo; così come dell'esistenza di umori diversi e, in particolare, di un latte alieno di buona qualità, di un latte proprio nocivo, di un latte alieno nocivo, di un latte proprio utile. Questa indicazione, pur nell'assenza di dettagli esplicativi, suggerisce l'idea che esistessero diversi tipi di latte con «destinatari» specifici.

latte umano (e non soltanto quello femminile!) potesse veicolare caratteri genetici, ci sembra particolarmente interessante la favola di Pollicino dei fratelli Grimm, in cui un gigante disceso dai monti rapisce al padre il minuscolo figlio per portarlo nella sua casa fra i boschi: facendolo poppare al suo seno, il piccolo bambino che non cresceva mai diventerà un essere gigantesco come il colossale padre «adottivo» (Lionetti parla di rito di iniziazione, motivando in questo modo il fatto che ad allattare il bambino non sia stata una gigantessa). Ricordiamo anche il caso di Thorgil, capo vichingo del X secolo, che, una volta perduta la moglie, riesce ad allattare personalmente il figlio, tagliandosi i capezzoli: per prima cosa sgorgò sangue, poi siero, infine latte. V.: Camporesi 1993, 12; Lionetti 1984, 19, 82-84. Cf. n. 81.

64 Alcune indicazioni relativamente alla nutrice in Aristotele (HA, VII 10, 587b: Tí Tөal, VII 12,

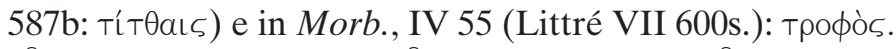

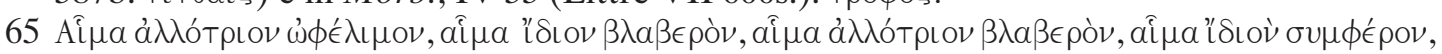

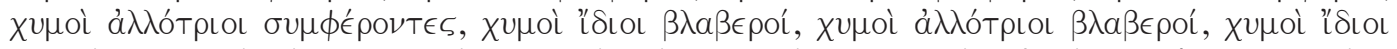

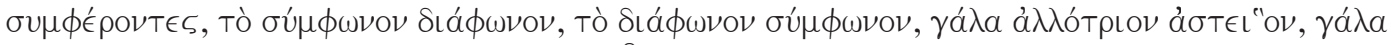

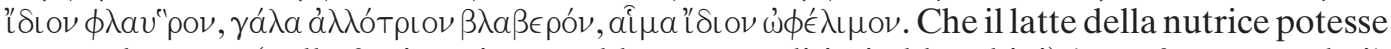
essere dannoso (nella fattispecie potrebbe causare litiasi al bambini) è confermato nel già citato passo ippocratico, v. n. precedente. 


\section{I diversi tipi di latte e la trasmissione di caratteri ereditari attraverso il latte umano nei testi letterari}

Questo paragrafo ha il medesimo scopo del precedente, ma attraverso l'uso di fonti di altra natura. Le fonti sono primarie, prevalentemente di epoca classica, con particolare attenzione per l'Orestea di Eschilo.

Pensiamo, poi, all'episodio dell'allattamento di Eracle da parte di Hera. Le fonti ci offrono un quadro assai complesso di questa «procedura» di adozione, all'interno della quale l'allattamento ha un ruolo significativo, perlomeno nel racconto di Diodoro (seppure con alcune significative puntualizzazioni). ${ }^{66}$

Ulteriori informazione possiamo ricavarle dai testi teatrali: Andromaca allatta i figli bastardi di Ettore (Eur., Andr., 224s.: il suo latte ha potere «nobilitante» e può rendere questi bambini illegittimi collactanei di Astianatte?); Giocasta allatta il nipote (Eur., $P h$., 986-89: il suo latte, in quanto sorella della defunta, è più adatto di altri?). ${ }^{67}$

Ma la testimonianza più pregnante ci sembra quella relativa al sogno di Clitemnestra,${ }^{68}$ «una delle metafore più forti e persistenti della colpa eredi-

66 Puntualizzazioni per le quali si rimanda a: Romani 2004, 94-97; Pirenne-Delforge 2010, 69195. Precisioni sono necessarie anche per quanto concerne la natura delle fonti: le fonti letterarie più antiche, che non risalgono oltre il IV secolo a.C., pongono questo avvenimento nella prima infanzia dell'eroe e alcune di esse sottolineano l'involontarietà della dea e talvolta anche la sua rabbia. Lo stesso tema si trova nelle fonti archeologiche - di epoca tarda e più spesso etrusche che greche - che, però, rappresentano sovente l'insolita immagine di un Eracle adulto e barbuto allattato dalla dea (v., a esempio: Bonfante 1989, 89; Bonfante 1997, 180). Le fonti sono: Diod. IV 9.6; Paus. IX 25.2; Lyc., Alex.,1327; Eratosth., Cat., XLIV; Hyg., Astr., II 43; Ach., Introd. In Aratum, XXIV (cf. Sch. Arat., 474 Maass); Gp., I A 19. Cf. anche $A P$, IX 589, in cui si descrive una statua che doveva sicuramente rappresentare una matrigna, dal momento che lo scultore non aveva messo latte nel suo seno «bastardo». Per Eracle che ferisce Hera al seno, v.: Hom., Il., V 392s.; Lyc., Alex., 39 (si noti che qui Hera viene de-

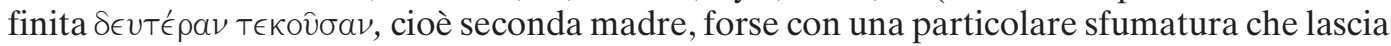
intendere madre per la seconda volta).

67 Ricordiamo soltanto in nota un epigramma di Ericio di Cizico (Antologia greca, VII 230), in cui una madre spartana afferma di avere nutrito codardi con il suo latte: quest'affermazione (I a.C.) potrebbe far pensare alla possibilità di trasmettere vizi e virtù col latte materno. Cf. Auberger 2010, 98.

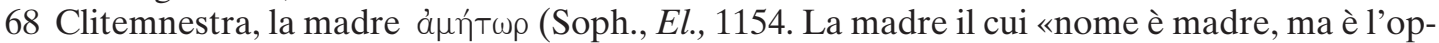
posto di una madre»: Soph., El., 1194, trad. U. Albini, Milano 1981; «la madre infernale che spira implacabile Ares contro i suoi cari»: Aeschl., Ag., 1235s.), allatta? La risposta non è così semplice. Clitemnestra allatta nelle tragedie di Euripide (Eur., IT, 231; IA, 1152: versione attestata per la prima volta, relativa a un figlio avuto da Tantalo, primo marito di Clitemnestra, ucciso da Agamennone assieme al figlio della coppia); ma il celebre passo in cui, nelle Coefore di Eschilo (vv. 896-98: «fermati, figlio, abbi ritegno, figlio mio, di questo seno, su cui tu spesso ti addormentavi succhiando con le gengive il latte che ben ti nutriva». Episodio ripreso in Eur., El., 1207) è stato interpretato come una negazione dell'allattamento del figlio. Secondo la Loraux 1991, 23s., il discorso della donna per intenerire il figlio, ricor-

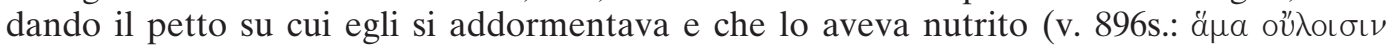


taria e dei rapporti familiari [...] nella trilogia tragica più complessa quanto a conflitti interni al genos». ${ }^{69}$ Clitemnestra ha sognato di partorire ( $\tau \in \kappa \in \hat{\imath} \nu$ ) un serpente, che ella poi avvolge nelle fasce come un bambino e che cerca nutrimento al suo seno. Ma al latte, che il piccolo serpente beve al seno della madre, si mescola un grumo di sangue, generato dal serpente stesso e causa per la donna di doloroso spavento (v. 533: «succhiò anzi nel latte un grumo di sangue $\left.{ }^{70}\right)$. Oreste comprende senza difficoltà il significato del sogno della madre: egli è il serpente allevato e nutrito da Clitemnestra ed è questo a fare di lui l'unico attore possibile della sua uccisione. È certo il figlio vendicatore di Agamennone, ma al tempo stesso è - come si coglie in maniera lampante in questo passo - il figlio di Clitemnestra: lei lo ha partorito, lei lo ha nutrito. E, dettaglio ancora più intrigante, quello che succhia è un $\theta \rho o ́ \mu \beta o \nu$, termine che, come ci insegna Chantraine, deriva «de la même base que тре́ $\phi \omega »$, che «avant de signifier nourrir a exprimé l'idée de faire grossir». ${ }^{71} \mathrm{Il}$ sangue, il latte, lo sperma e il processo di formazione di un individuo sembrano emergere, con un sottile gioco lessicale e semantico, intrecciati in maniera magistrale.

\section{Latte, seno e follia}

E, forse, c'è anche di più: "faut-il voir dans la présence du sang dans les mamelles un signe de folie? $\gg^{72}$ Ricordiamo, a questo proposito, un aforisma

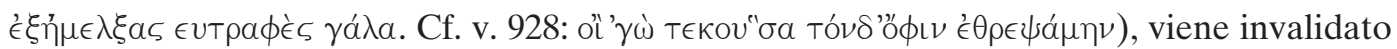
in anticipo da quello della nutrice, che afferma di aver ricevuto dal padre il bambino (v. 762:

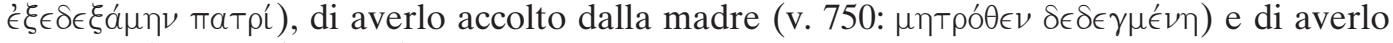
allevato (v. 750: $\dot{\epsilon}^{\prime} \dot{\epsilon}^{\prime} \theta \rho \in \psi \alpha$ ). La nutrice di Oreste - l'unica «realistica» nella tragedia greca (v. Capomacchia 1994) - afferma di avere esattamente quelle mansioni che noi ci aspetteremmo per una трофоєús (come ella stessa si definisce, v. 760): cambiare il bambino, lavare le sue fasce, vegliarlo di notte. Ma da queste parole non possiamo dedurre se spettasse a lei anche allattarlo. È verosimile che il bambino di notte dormisse separatamente con le ancelle, ma che la madre si svegliasse per dargli il latte (cf. Lys., 1, 9s.). Il sogno del parto del serpente, cui Clitemnestra porge il seno pensando di avere a che fare con un bambino, sembra confermare il fatto che la donna allattasse personalmente. Il dettaglio del grumo di sangue succhiato con il latte (vv. 533, 546) anticipa e prepara la negazione della parentela che verrà esplicitata nelle Eumenidi (vv. 606-08): alla domanda di Oreste «e io sarei dello stesso sangue di mia madre?», il coro risponde «e come, dunque, ti nutrì ( $\left.{ }^{\prime} \theta \rho \in \psi \in \nu\right)$, o scellerato, nel suo seno? Tu rinneghi il dolcissimo sangue di tua madre?».

69 Bindi 1999, 86.

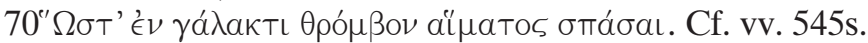

71 Chantraine 1968, s.u. $\theta$ pómßos. Cf. Demont 1978, 359. Cf. anche n. 38. Un verbo formato

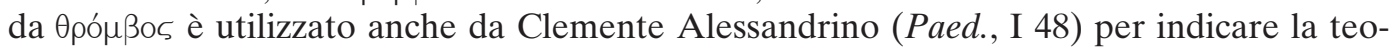
ria del concepimento e della formazione del feto, come osserva Demont 1978, 381s. Clemente conosceva (e condivideva), come abbiamo visto, la teoria dell'emogenesi del latte, v. nn. 9, 58 .

72 Dumortier 1975, 22. 


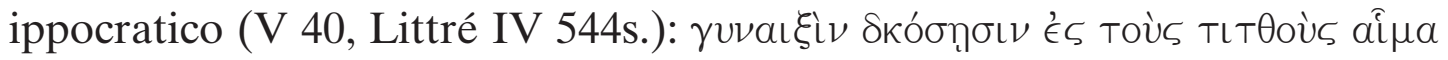

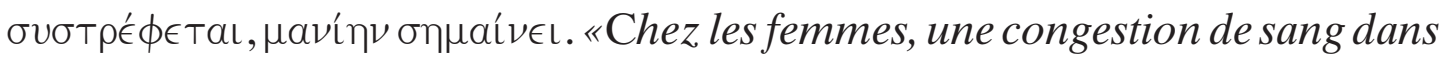
les mamelles annonce la folie. $\gg^{73}$

Esiste nei testi medici greci un singolare legame fra seno e pazzia. Era credenza, infatti, che nei seni si trovassero due vene ${ }^{74}$, nelle quali si sarebbero potute accumulare grandi quantità di sangue, che avrebbero potuto causare comportamenti bizzarri e sconvenienti. Quest'eventualità si sarebbe potuta verificare più facilmente in caso di assenza di mestruazioni, grazie alle quali mensilmente la donna si «purgava»; purga che, durante l'allattamento, essendo il latte sangue mestruale cotto, non poteva che avvenire attraverso l'espulsione del latte dai seni. L'isteria, la più tipica malattia femminile, trova origine proprio nell'incepparsi di questa meccanica dei fluidi, che è alla base della fisiologia muliebre, dovuta agli spostamenti dell'utero, animale irre-

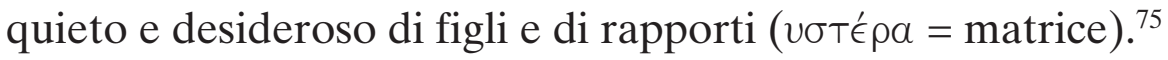

La causa della follia muliebre va sovente rintracciata in un ingorgo causato da una massa ematica. È il caso soprattutto delle vergini, nel cui corpo «chiuso» il sangue in eccesso del menarca non riesce a trovare un'adeguata via di sbocco, ma anche delle donne anziane, ormai prive di quella salutare attività sessuale che favorisce il fisiologico fluire degli umori femminili. ${ }^{76}$ Dopo il parto, la donna è meno esposta a questo tipo di «soffocamento ematico», in quanto il suo corpo diviene meglio predisposto a far defluire il sangue mestruale, a causa della dilatazione che le vene e l'utero hanno subito durante il travaglio (Mul., I 1, Littré VIII 8-13). L'allattamento, come le altre normali attività fisiologiche femminili (il coito, la maternità, il parto, le mestruazioni), è concepito come funzione terapeutica di questo immaginario mal d'être femme, ${ }^{77}$ quindi come attività salutare e raccomandabile per la donna. Durante l'allattamento, però, si possono non di rado verificare ma-

73 Cf. Ep., II 32 (Littré V 138).

74 Vene che, peraltro, avrebbero contenuto la più grande porzione di intelligenza (Ep., II 19, Littré V 136). È interessante notare che, stando a una testimonianza di Sorano (III 3), esiste un legame simpatetico fra le meningi e l'utero (causa della perdita delle inibizioni, v. Marzari 2010, 61) e che, secondo una diffusa e duratura credenza, esiste un collegamento, anzi, una perfetta corrispondenza, tra utero e seno, grazie al quale il sangue sarebbe passato dal primo al secondo, divenendo latte. Soltanto alcuni esempi per quest'ultimo punto: Mul., II 174, Littré VII 354; Gal., IV 179 K.; e ancora in Clem., Paed., I 39. V.: Pomata 1995, 48; Bodiou 2006, 165; Tognazzi 2008.

75 Si fa riferimento, ovviamente, al noto passo platonico: Tim., 91c. Cf. Aret., SA, II 11. Per l'isteria, termine medico che è stato utilizzato nel corso dei secoli per indicare malattie con sintomatologie significativamente differenti fra loro, v. Pigeaud 1987, 11s., 83.

76 Marzari 2010, 50-52. L'anatomo-fisiologia del corpo femminile è pensata secondo una scansione fondamentale di chiusura-apertura, v. Sissa 1992.

77 Manuli 1983, 152. 
stiti, cioè «ingorghi di latte» (quindi ingorghi a tutti gli effetti «ematici», stando alle teorie mediche antiche), che potrebbero spiegare questo singolare e straordinario legame fra seno (allattamento) e pazzia presente nel Corpus Hippocraticum.

\section{Il contributo dell'iconografia}

In relazione alla derivazione del latte materno dal sangue mestruale, vorremo proporre anche alcune riflessioni per quanto concerne la quasi totale assenza di scene di allattamento nell'iconografia greca di epoca arcaica e classica, argomento su cui si è a lungo dibattuto, ma relativamente al quale non sono mai state fornite, a nostro avviso, spiegazioni convincenti ed esaustive. È stato a più riprese osservato - a nostro avviso erroneamente - che l'assenza di immagini di donne che allattano stupisce, soprattutto in relazione all'abbondanza di scene di vita quotidiana e di intimità fra donne e bambini. ${ }^{78}$ «Per i ceramografi greci» - scrive, al contrario, Lissarrague - «la maternità non è un tema iconografico pertinente». ${ }^{79}$ Lo studioso francese fa esplicito riferimento soltanto ai ceramografi, argomentando che sulle stele funerarie di fine V-IV secolo a.C. (ci stiamo, però, già avvicinando all'epoca ellenistica) le scene «materne» sono piuttosto numerose. Per spiegare questa discrepanza, egli fa riferimento alla diversità di funzioni: le immagini sui vasi, più generiche, non dovrebbero rappresentare individui particolari, ma evocare modelli mitici o momenti esemplari della vita sociale. Lissarrague, a sostegno delle sue affermazioni, non si limita a prendere in considerazione scene di donne in compagnia di bambini, ma anche scene di parto e di allattamento. A con-

78 Bonfante 1989, 64; Bonfante 1997, 175.

79 Lissarrague 1997, 209. L'eccezione è assai eloquente: sopra un'idra del 490 a.C. circa troviamo quella che a prima vista potrebbe essere una scena idilliaca: sulla sinistra un uomo barbato di profilo destro, appoggiato al suo bastone, osserva una donna di profilo destro seduta che offre il seno a un bambino di profilo sinistro che sta sulle sue ginocchia; ai suoi piedi, due galli uno di fronte all'altro; sulla destra una donna che fila di profilo sinistro, in piedi vicino a un cesto per la lana. In questa immagine possiamo apprezzare una scena di vita familiare: genitori, figlio e una serva sono serenamente riuniti. Le iscrizioni che accompagnano questi personaggi confermano i rapporti di parentela, rivelando però un significato mitologico che trasforma radicalmente l'immagine. Il padre è Anfiarao, la madre è Erifile e il figlio è Alcmeone. Anfiarao, re di Argo, tradito dalla moglie morirà tentando di ristabilire Polinice sul trono di Tebe; Alcmeone ucciderà sua madre per vendicare il padre. Si comprende pertanto tutta l'ironia tragica di quest'immagine, che, ben lungi dall'illustrare l'incanto della vita familiare, evoca implicitamente una serie di dissidi; in questo modo si comprende meglio, inoltre, la presenza del combattimento fra galli, che ricorda la lotta fratricida tra Eteocle e Polinice e mostra, secondo Lissarrague 1997, 211, tav. VIII, nel cuore dell'oikos la discordia e l'invidia che dividono famiglie e città. 
clusione delle nostre ricerche, vorremmo correggere leggermente l'affermazione dello studioso francese, dicendo che le scene di maternità per così dire «esplicita» non vengono considerate un tema iconografico pertinente. Se le immagini di madri non sono particolarmente numerose, infatti, sono comunque assai più frequenti rispetto a quelle di parto, di allattamento e di donne incinte: in questi ultimi casi ci pare maggiormente opportuno parlare di temi iconografici non pertinenti ovvero non «congeniali», non particolarmente gradevoli (soprattutto nel caso dell'allattamento, considerando l'emogenesi del latte materno), di «faccende di donne» - per usare le parole di Lissarrague - che si evita di rappresentare. Oppure, forse ancor più banalmente, di tematiche prive di interesse per gli $\operatorname{artisti}^{80}$ (che, ricordiamo, di norma erano maschi e che, verosimilmente, nutrivano scarsa «curiosità» per il funzionamento biologico del corpo femminile).

\section{Conclusioni}

Dall'analisi delle fonti proposte in questa sede, assai differenti fra loro per contenuti, epoca e difficoltà interpretative, ci pare di poter concludere che nella Grecia arcaica e classica, come a Roma, erano significativamente radicati i pregiudizi nei confronti del sangue mestruale. La teoria dell'emogenesi del latte umano (ampiamente enunciata nei trattati ippocratici e talmente radicata da essere ripresa non soltanto, a esempio, da Clemente Alessandrino, ma persino da giungere, attraverso molteplici strade e con diversi piani di lettura, fino ai nostri giorni ${ }^{81}$ ) a nostro avviso non può non avere considerevolmente influenzato, in senso negativo, l'atteggiamento dell'uomo sia nei confronti del latte animale (e, almeno in parte, dei suoi derivati), sia nei confronti di quello umano. Questo disagio si traduce anche a livello iconografico, in particolare per quanto concerne la quasi totale assenza di immagini di donne che allattano in Attica in epoca arcaica e classica, assenza di

80 Lissarrague 1997, 209, anche in questo caso si limita a parlare di pittori.

81 Eco di simili credenze troviamo, a esempio, ancora in epoca moderna: Camporesi 1993, 20s., che sta parlando di vampirismo presso i Morlacchi, scrive (cf. n. 63): Il vampirismo infatti rappresenta il volto truculento e sinistro, l'immagine rovesciata ma speculare di quel tabù del sangue umano praticato da molte culture (fra cui l'ebraica), che fra i sacerdoti delle tribù allobroghe del Delfinato e della Savoia sui quali gravava il divieto non soltanto di spargerlo e di berlo, ma anche di toccarlo, era stato formalizzato il precetto di sottrarre i bimbi destinati al sacerdozio all'allattamento materno perché il latte altro non era, raccontava il vescovo francescano Antonio de Guevara nel Libro di Marco Aurelio offrendo alle principesse di Spagna un ricco campionario di forme superstiziose e pagane di allattamento, che bevere sangue bianco, perché la latta bianca altro non è che sangue cotto, e il sangue colorito altro non è che latta cruda. 
solito attribuita al fatto che le donne greche non allattavano personalmente i figli (ma smentita da un'analisi più attenta delle fonti). ${ }^{82}$

\section{Bibliografia}

Albertocchi, Marina, «〈Eugenie $>$ ebbre? Considerazioni su alcune pratiche rituali del Thesmophorion di Bitalemi a Gela», Kernos 25 (2012) 57-74

Auberger, Janick, Manger en Grèce classique. La nourriture, ses plaisirs et ses contraintes (Québec 2010)

Bettini, Maurizio, Nascere. Storie di donne, donnole, madri ed eroi (Torino 1998)

Bindi, Letizia, La tragedia in corpo. Luoghi dell'identità greca (Reggio Calabria 1999)

Bodiou, Lydie, «De l'utilité du ventre des femmes. Lectures médicales du corps féminin», in: Francis Prost/Jérôme Wilgaux (éds.), Penser et représenter le corps dans l'Antiquité, Actes du colloque international de Rennes, 1-4 septembre 2004 (Rennes 2006) 153-66

Bodiou, Lydie, «Les singulières conversions du lait maternel à l'époque classique. Approche médicale et biologique», Pallas 85 (2011) 141-51

Bonnet, Valérie, «Les animaux occidentaux dans la pharmacopée de Pline», in: Homme et animal dans l'antiquité romaine. Actes du colloque de Nantes 1991 (Tours 1995) 163-72

Bonfante, Larissa, «Iconografia delle madri: Etruria e Italia antica», in Antonia Rallo (cur.), Le donne in Etruria (Roma 1989) 85-106

Bonfante, Larissa, "Nursing Mothers in Classical Art", in: Ann Olga Koloski/Claire L. Lyons (eds.), Naked Truths. Women, Sexuality, and Gender in Classical Art and Archaeology (London-New York 1997) 174-96

Camporesi, Piero, Le vie del latte. Dalla Padania alla steppa (Milano 1993).

Capomacchia, Anna Maria Gloria, «Nutrice di eroi: ruolo e valenza di un personaggio <minore〉 nella tragedia greca», Studi e Materiali di Storia delle Religioni 18 (1994) 11-24

Caratozzolo, Antonino, Corpo umano e contenitori. Ricerche sul lessico vascolare greco, Tesi di dottorato (Siena 2007)

Chantraine, Pierre, Dictionnaire etymologique de la langue grecque. Histoire des mots (Paris 1968, ripr. Paris 1999)

Cicala, Valeria, «Appunti su alcuni impieghi del latte nell'antichità», in: Tozzi Fontana-Montanari 2000, 37-42

Dalby, Andrew, Siren Feasts. History of Food and Gastronomy in Greece (LondonNew York 1996)

Dalby, Andrew, Food in the Ancient World from A to Z (New York 2003)

Danese, Roberto M., «Lac humanum fellare. La trasmissione del latte e la linea della generazione», in: Renato Raffaelli/Roberto M. Danese/Settimio Lanciotti (curr.), Pietas e allattamento filiale. La vicenda, l'exemplum, l'iconografia. Colloquio di Urbino, 2-3 maggio 1996 (Urbino 1997) 39-72

82 Pedrucci 2013, in cui spiega che le ragioni sono verosimilmente da connettere, oltre che con l'emogenesi del latte, con il concetto di aidos. 
Danese, Roberto M. et alii (curr.), Allattamento filiale. La fortuna. Colloquio di Urbino, 28-29 aprile 1998 (Urbino 2000)

Dasen, Véronique, «Des nourrices grecques à Rome?», in: Véronique Pache-Huber, Véronique Dasen (eds.), Politics of Child Care in Historical Perspective. From the World of Wet Nurses to the Networks of Family Child Care Providers (special issue of Paedagogica Historica 46, 2010), 699-713 (http://dx.doi.org/10.1080/00309230. 2010.526330)

Davidson, Hilda Ellis, "Milk and the Northern Goddesss", in: Sandra Billington/ Miranda Green (eds), The Concept of the Goddess (London-New York 1996) 91-106

Dean-Jones, Lesley, Women's Bodies in Classical Greek Science (Oxford 1994)

De Grossi Mazzorin, Jacopo/Minniti, Claudia, «L'utilizzazione degli animali nella documentazione archeozoologica a Roma e nel Lazio dalla preistoria recente all'età classica», in: Luciana Drago Troccoli (ed.), Il Lazio dai Colli Albani ai Monti Lepini tra preistoria ed età moderna (Roma 2010) 39-67

Deichgräber, Karl, «Zur Milchtherapie der Hippokratiker (Epid. VII)», in: HansHeinz Eulner et alii (eds.), Medizingeschichte in unserer Zeit (Stuttgart 1971) $36-53$

Delaini, Paolo, «Le corps animal comme médicament chez les Yaghnobis», in: Antonio Panaino-Andrea Piras (eds.), Studi Iranici Ravennati I (Milano 2011) 159-68

Delatte, Armand, Le cycéon, breuvage rituel des mystères d'Eleusis (Paris 1954)

Demont, Paul, «Remarques sur le sens de трє́ $\psi(\omega »$, Revue des études grecques 91 (1978) 358-84

Djéribi, Muriel, «Le Mauvais œil et le lait», L’homme 105 (1988) 35-47

Dodds, Eric Robertson, I Greci e l'irrazionale (Milano 2003). Ed. or.: The Greeks and the Irrational (Berkeley-Los Angeles 1951)

DuBois, Page, Il corpo come metafora. Rappresentazioni della donna nella Grecia antica (Roma-Bari 1990). Ed. or.: Showing the Body (London 1988)

Dumortier, Jean, Le vocabulaire médical d'Eschyle et les écrits hippocratiques (Paris 1975)

Franco, Cristiana, Senza ritegno. Il cane e la donna nell'immaginario della Grecia antica (Bologna 2003)

Frontisi-Ducroux, Françoise/Vernant, Jean-Pierre, Ulisse e lo specchio. Il femminile e la rappresentazione di sé nella Grecia antica (Roma 2003). Ed. or.: Dans l'œil du miroir (Paris 1997)

Gordon, Ian R., Controlled Reproduction in Sheep and Goats, Wallingford 1997

Gourevitch, Danielle, «Le chien, de la thérapeutique populaire aux cultes sanitaires», Mélanges d'Archéologie et d'Histoire de l'Ecole Française de Rome, Antiquité 80 (1968) 247-81

Gourevitch, Danielle, Le mal d'être femme. La femme et la médecine dans la Rome antique (Paris 1984)

Gualerzi, Saverio, Penelope o della tessitura. Trame femminili da Omero a Ovidio (Bari 2007)

Harris, Marvin, Buono da mangiare. Enigmi del gusto e consuetudini alimentari (Torino 1990). Ed. or.: Good to Eat. Riddles of Food and Culture (New York 1985) 
Hartog, François, Memoria di Ulisse. Racconti sulla frontiera nell'antica Grecia (Torino 2002). Ed. or.: Mémoire d'Ulysse. Récits sur la frontière en Grèce ancienne (Paris 1996)

Khatib-Chahidi, Jean, «La parentela di latte nell'Iran musulmano sciita», in: Maher 1992, 119-41

King, Helen, "Sacrificial Blood: The role of the Amnion in Ancient Gynecology", in: Marilyn Skinner (ed.), Rescuing Creusa. New Methodological Approaches to Women in Antiquity (Lubbock 1987) 117-25

King, Helen, Hippocrates' Woman. Reading the Female Body in Ancient Greece (London-New York 1988)

Laskaris, Julie, "Nursing Mothers in Greek and Roman Medicine", American Journal of Archaeology 112 (2008) 459-64

Lévi-Strauss, Claude, Il totemismo oggi (Milano 1991). Ed. or.: Le totémisme aujourd'hui (Paris 1962)

Lionetti, Roberto, Latte di padre (Brescia 1984)

Lissarrague, François, «Uno sguardo ateniese», in: Georges Duby-Michelle PerrotPauline Schmitt Pantel (curr.), Storia delle donne. L'Antichità (Roma-Bari 1997), 179-240. Ed. or. Histoire des femmes en Occident. L'Antiquité (Paris 1991)

Loraux, Nicole, Il femminile el'uomo greco (Roma-Bari1991). Ed. or.: Les expériences de Tirésias. Le féminin et l'homme grec (Paris 1989)

Maher, Vanessa (cur.), Il latte materno. I condizionamenti culturali di un comportamento (Torino 1992)

Manuli, Paola, «Donne mascoline, femmine sterili, vergini perpetue: la ginecologia greca tra Ippocrate e Sorano», in: Silvia Campese/Paola Manuli/Giulia Sissa (eds.), Madre materia. Sociologia e biologia della donna greca (Torino 1983) 149-204

Marzari, Francesca, «Paradigmi di follia e lussuria virginale in Grecia antica: le Pretidi fra tradizione mitica e medica», I Quaderni del Ramo d'Oro on-line 3 (2010) 47-74 (http://www.qro.unisi.it/frontend/node/71)

Matthey, Piero, «Il 〈milk complex〉 fra i Bantu orientali», in: Oddone Longo/Paolo Scarpi (curr.), Homo edens. Regimi, miti e pratiche dell'alimentazione nella civiltà del Mediterraneo. Atti del Congresso tenuto a Verona nel 1987 (Milano 1989) 49-55

Mauss, Marcel, Teoria generale della magia e altri saggi (Torino 1965). Ed. or.: Sociologie et anthtropologie (Paris 1950)

Molinos Tejada, María Teresa, «Las nodrizas en la escena clásica», in: Francesco De Martino/Carmen Morenilla Talens (eds.), El Fil d'Ariadna. Universitat de València 3-5 de maig 2000 (Bari 2001) 299-316

Molinos Tejada, María Teresa, «Madres y nodrizas en la Antigüedad», in: Marta González González/M. Amparo Pedregal Rodríguez (eds.), Venus sin espejo. Imágenes de mujeres en la Antigüedad clásica y el cristianismo primitivo (Oviedo 2005) 57-79

Momigliano, Arnaldo, «Le regole del giuoco nello studio della storia antica», in: Decimo contributo alla storia degli studi classici e del mondo antico (Roma 1980) $13-22$

Montanari, Massimo, «Il latte e i suoi derivati nella tradizione alimentare italiana», in: Tozzi Fontana-Montanari 2000, 9-36

Montanari, Massimo, «Il latte fra natura e cultura», in: G. Costeo, Sulla natura del latte (Torino 2001) 9-11 
Naso, Irma, Formaggi del Medioevo: la «Summa lacticiniorum» di Pantaleone da Confienza (Torino 1990).

Noakes, David E. et alii, Ostetricia e riproduzione veterinaria (Bologna 2008). Ed. or.: Arthur's Veterinary Reproduction and Obstetrics, Elsevier Health Sciences 2001

Parkes, Peter, "Milk Kinship in Islam. Substance, Structure, History", Social Anthropology 13 (2005) 307-329

Pedrucci, Giulia, L'allattamento nella Grecia di epoca arcaica e classica (Roma 2013)

Pigeaud, Jackie, «La présure et le lait. Quelques remarques sur la rêverie de la caille du lait», Les Etudes Classiques 42 (1975) 3-17

Pigeaud, Jackie, Folie et cures de la folie chez les médecins de l'antiquité gréco-romaine. La manie (Paris 1987)

Pirenne-Delforge, Vinciane, «Nourricières d'immortalité: Déméter, Héra et autres déesses en pays grec», Paedagogica Historica 46.6 (2010) 685-97 (http://dx.doi. org/10.1080/00309230.2010.526328)

Pomata, Gianna, «La «meravigliosa armonia〉. Il rapporto fra seno ed utero dall'anatomia vascolare all'endocrinologia», in: Giovanna Fiume (cur.), Madri. Storia di un ruolo sociale (Venezia 1995) 45-81

Prêtre, Clarisse/Charlier, Philippe, Maladies humaines, thérapies divines. Analyse épigraphique et paléopathologique de textes de guérison grecs (Villeneuve-d'Ascq 2009)

Quevedo Sánchez, Alejandro, «Hayes 121 e 123. Due forme poco frequenti di TSA A documentate a Carthago Nova», in: L'Africa Romana XVIII, Olbia 2008 (Roma 2010) 2071-82

Rey, André-Louis, «Autour des nourrissons byzantins et de leur régime», in: Véronique Dasen (éd.), Naissance et petite enfance dans l'antiquité. Actes du colloque de Fribourg, 28 novembre-1 ${ }^{\text {er }}$ décembre 2001 (Fribourg-Göttingen 2004) 363-75

Romani, Silvia, Nascite speciali. Usi e abusi del modello biologico del parto e della gravidanza nel mondo antico (Alessandria 2004)

Sissa, Giulia, La verginità in Grecia (Roma-Bari 1992). Ed. or.: Le corps virginal. La virginité féminine en Grèce ancienne (Paris 1987)

Stenson, Garry B., "Oestrus and the Vaginal Smear Cycle of the River Otter, Lutra Canadensis", J. Reprod. Fertil. 83 (1988) 605-10

Théodoridés, Jean, Histoire de la rage. Cave canem (Paris 1986)

Tognazzi, Giada, «La concezione biologica della donna nel Corpus Hippocraticum: dalla teoria alla terapia», Genre et Histoire 2 (2008) (http://genrehistoire.revues. org/index331.html)

Tozzi-Fontana, Massimo/Montanari, Massimo (curr.), Il latte. Storia, lessici, fonti (Bologna 2000)

Triplett, Katja, "Esoteric Buddhist Eye-healing Rituals in Japan and the Promotion of Benefits", in: Axel Michaels (ed.), Ritual Dynamics and the Science of Ritual (Wiesbaden 2010) 485-99

Vilatte, Sylvie, «La femme, l'esclave, le cheval et le chien: les emblèmes du kalos kagathos Ischomaque», Dialogues d'histoire ancienne 12 (1986) 271-94

von Staden, Heinrich, "Women and dirt", Helios 19 (1992) 7-29 